\title{
Perspective
}

Actualité en histoire de l'art

\section{Archéologie et religiosité des peuples italiques sur les territoires situés entre les mers Adriatique, Ionienne et Tyrrhénienne}

Archeology and Religiosity of the Italic Peoples in the Territories Situated

Between the Adriatic, Ionian and Tyrrhenian Seas

Archäologie und Religiosität der italischen Völker in den Gebieten zwischen dem adriatischen, ionischen und tyrrhenischen Meer

Archeologia e religiosità dei popoli italici nei territori fra Adriatico, Ionio e

Tirreno

Arqueología y religiosidad de los pueblos itálicos de los territorios situados entre los mares Adriático, Jónico y Tyrrhénico

\section{Angelo Bottini}

\section{(2) OpenEdition}

\section{Journals}

Édition électronique

URL : https://journals.openedition.org/perspective/7121

DOI : 10.4000/perspective. 7121

ISSN : 2269-7721

Éditeur

Institut national d'histoire de l'art

Édition imprimée

Date de publication : 30 juin 2017

Pagination : 65-90

ISBN : 9782917902387

ISSN : $1777-7852$

Référence électronique

Angelo Bottini, « Archéologie et religiosité des peuples italiques sur les territoires situés entre les mers Adriatique, Ionienne et Tyrrhénienne », Perspective [En ligne], 1 | 2017, mis en ligne le 31 décembre 2017, consulté le 16 janvier 2023. URL : http://journals.openedition.org/perspective/7121 ; DOI : https://doi.org/10.4000/perspective.7121 


\section{TRAVAUX}

Angelo Bottini

\section{Archéologie et religiosité des peuples italiques sur les territoires situés entre les mers Adriatique, Ionienne et Tyrrhénienne}

La religiosité constitue, à n'en pas douter, l'un des phénomènes les plus complexes et les plus structurés développés par les différentes cultures. Elle put se manifester sous l'aspect d'œuvres spectaculaires et de la plus haute importance, résultant du travail durable de communautés entières - on pense aussitôt, dans le cas du monde méditerranéen antique, aux temples agrémentés d'un riche appareil décoratif et aux sanctuaires, avec leurs mobiliers composés d'offrandes variées - ou bien animer au contraire des comportements individuels ou tout au plus circonscrits à un noyau familial, ou à un groupe d'individus liés par des activités, des croyances et des dévotions.

De tout cela, l'archéologie ne peut saisir que les objets ayant pris la forme d'entités matérielles susceptibles de traverser les siècles : des édifices, des installations et des objets isolés dont l'attribution à la sphère religieuse est rendue possible, entre autres, par l'acquisition progressive d'une connaissance globale de la culture qui les a produits. Si, au XVIII siècle, on a pu désigner par le terme de basilique, au sens romain, le plus ancien temple dorique de Poseidonia/Paestum, parce qu'on le considérait comme tel, on connaît aujourd'hui si bien la forme, la structure et l'aspect des monuments grecs, mais aussi étrusco-italiques et romains, appartenant à cette sphère, que l'on peut reconstituer par la pensée la totalité d'un édifice à partir d'un ensemble d'éléments contingents maintenant désarticulés, voire à partir de ses traces en négatif. Étudiées par un chercheur possédant une connaissance adéquate du sujet, les fosses de déprédation des blocs de fondation permettent, par exemple, de reconstituer dans ses

Angelo Bottini a été Soprintendente archéologue de la Basilicate, de la Toscane et enfin de Rome. Il a enseigné l'archéologie de la Grande Grèce et des peuples italiques à Florence, à Sienne et à Matera (Università della Basilicata). Il est l'auteur de 150 travaux de recherche scientifique, principalement consacrés à I'Italie méridionale préromaine. 
1. Carte des sites archéologiques cités.

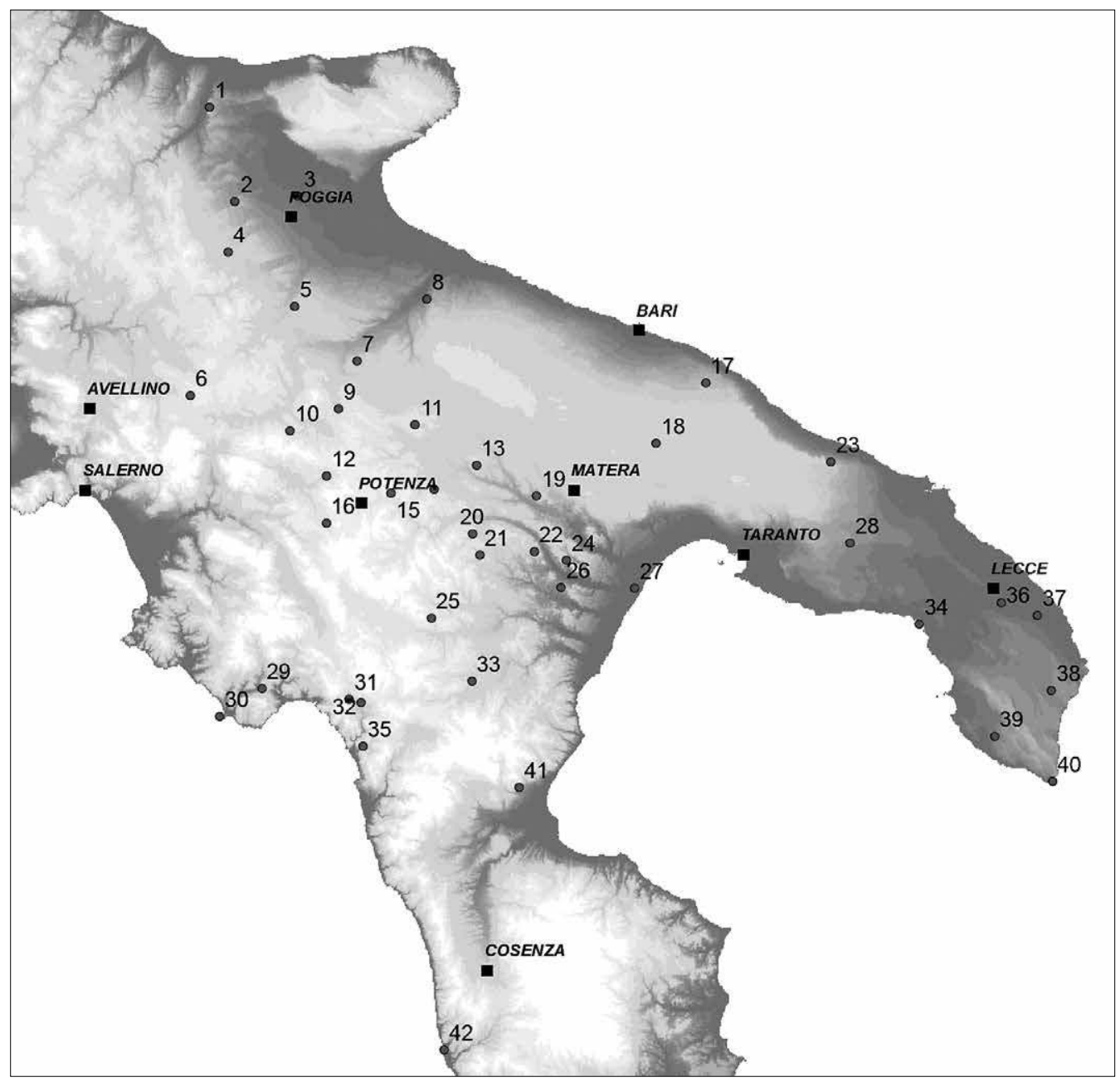

grandes lignes le plan originel d'un édifice. Une telle pratique permet d'aboutir à des résultats d'une importance capitale, à la condition de prendre soin de respecter le contexte historique et culturel auquel on se réfère, sous peine de commettre de graves erreurs et contresens.

Dans la seconde moitié des années 1980, l'analyse méticuleuse des nombreuses terres cuites architecturales d'époque archaïque découvertes à Braida del Vaglio, en Basilicate, était encore orientée vers la reconstitution d'un temple grec; et ce sous l'effet de la conviction, aussi préconçue qu'infondée, selon laquelle l'adoption par les indigènes des systèmes décoratifs des toitures helléniques impliquait systématiquement l'érection d'édifices de culte "publics " (LO PORTO, RANALDI, 1990 ; avec une nouvelle hypothèse de reconstitution dans GRECO, 2011 ; voir aussi SETARI, 2009). Aujourd'hui, grâce aux remarquables résultats des fouilles menées sur le site proche de Torre di Satriano, nous savons avec certitude qu'à Vaglio, comme sur d'autres sites jusqu'ici moins connus, on observe au contraire des ensembles architecturaux auxquels Massimo Osanna a proposé de donner le nom d'anaktoron, afin d'en souligner la fonction principale de résidence d'un groupe dominant. Au sein de ces ensembles pouvaient sans aucun doute se tenir, comme on le verra plus loin, des activités à caractère collectif (Segni del potere..., 2013). 


\begin{tabular}{|c|c|c|c|}
\hline site antique / localité (commune) & province & région & numéro \\
\hline Arpi (Foggia) & Foggia & Pouilles & 3 \\
\hline Ascoli Satriano & Foggia & Pouilles & 5 \\
\hline Banzi & Potenza & Basilicate & 11 \\
\hline Campora San Giovanni (Amantea) & Cosenza & Calabre & 42 \\
\hline Canosa & Barletta, Andria, Trani & Pouilles & 8 \\
\hline Cavallino & Lecce & Pouilles & 36 \\
\hline Chiaromonte & Potenza & Basilicate & 33 \\
\hline Conversano & Bari & Pouilles & 17 \\
\hline Ferrandina & Matera & Basilicate & 22 \\
\hline Francavilla Marittima & Cosenza & Calabre & 41 \\
\hline Garaguso & Matera & Basilicate & 20 \\
\hline Irsina & Matera & Basilicate & 13 \\
\hline Lavello & Potenza & Basilicate & 7 \\
\hline Lucera & Foggia & Pouilles & 2 \\
\hline Metaponto (Bernalda) & Matera & Basilicate & 27 \\
\hline Monte Sannace (Gioia del Colle) & Bari & Pouilles & 18 \\
\hline Nemoli & Potenza & Basilicate & 32 \\
\hline Oria & Brindisi & Pouilles & 28 \\
\hline Ostuni & Brindisi & Pouilles & 23 \\
\hline Palinuro & Salerno & Campanie & 30 \\
\hline Pisticci & Matera & Basilicate & 26 \\
\hline Pomarico Vecchio (Pomarico) & Matera & Basilicate & 24 \\
\hline Porto Cesareo & Lecce & Pouilles & 34 \\
\hline Ripacandida & Potenza & Basilicate & 9 \\
\hline Rivello & Potenza & Basilicate & 31 \\
\hline Rocavecchia (Melendugno) & Lecce & Pouilles & 37 \\
\hline Roccagloriosa & Salerno & Campanie & 29 \\
\hline Ruoti & Potenza & Basilicate & 12 \\
\hline Ruvo del Monte & Potenza & Basilicate & 10 \\
\hline San Chirico Nuovo & Potenza & Basilicate & 14 \\
\hline San Mauro Forte & Matera & Basilicate & 21 \\
\hline Santa Maria di Leuca (Castrignano del Capo) & Lecce & Pouilles & 40 \\
\hline Serra Lustarnte (Armento) & Potenza & Basilicate & 25 \\
\hline Tiati (San Paolo Civitate) & Foggia & Pouilles & 1 \\
\hline Timmari (Matera) & Matera & Basilicate & 19 \\
\hline Tortora & Cosenza & Calabre & 35 \\
\hline Torre di Satriano (Tito) & Potenza & Basilicate & 16 \\
\hline Troia & Foggia & Pouilles & 4 \\
\hline Ugento & Lecce & Pouilles & 39 \\
\hline Valle d'Ansanto (Rocca San Felice) & Avellino & Campanie & 6 \\
\hline Vaste (Poggiardo) & Lecce & Pouilles & 38 \\
\hline Vaglio Basilicata & Potenza & Basilicate & 15 \\
\hline
\end{tabular}


De manière plus générale, une évidence apparaît désormais : pendant toute l'époque archaïque, du VII ${ }^{\mathrm{e}}$ au $\mathrm{V}^{\mathrm{e}}$ siècle avant notre ère, au sein des populations installées sur les territoires méridionaux de la Péninsule italienne, véritable mosaïque de cultures, de langues et donc sans doute aussi d'origines diverses, l'interaction, parfois précoce et intense, avec les diverses poleis grecques établies sur les côtes (mais aussi, au moins pour une partie non négligeable des territoires en question, avec les centres étrusques de la région tyrrhénienne), est restée la prérogative des élites. Elle n'a donc pas eu pour conséquence immédiate le dépassement de modèles d'organisation sociale d'origine protohistorique, ou encore l'adoption intégrale et homogène de comportements caractéristiques des communautés urbaines. Le cas de l'armement de type hoplitique est, de ce point de vue, emblématique : adopté presque partout, il demeura cependant toujours l'apanage d'une minorité restreinte qui l'exhibait, tout au long de la vie et dans la mort, sans la moindre référence à la tactique de combat qui en avait causé l'apparition.

Pour revenir aux formes de religiosité, et même si les généralisations sont toujours des plus dangereuses, aucune figure divine à qui l'ensemble de la communauté se serait identifiée, et à laquelle on aurait donc attribué le droit de posséder une "maison " - un naos -, aussi reconnaissable que prestigieuse, n'émerge particulièrement. On ne perçoit pas non plus la nécessité de " marquer " de la même façon des lieux-clef du territoire qui placeraient sous la protection de tel ou tel dieu les activités qui s'y déroulaient, ou qui feraient de ce même dieu le gardien de ses frontières. Autant de phénomènes que l'on observe en revanche, ponctuellement, dans le cas des poleis achéennes installées sur les rives des mers Ionienne et Tyrrhénienne (GRECO, 2016, p. 206). Cela n'exclut pas pour autant que la religiosité ait pu représenter un aspect significatif de la vie des communautés en question, et qu'elle ait contribué que dans une moindre mesure à en définir les mentalités et les pratiques, individuelles ou collectives. Il convient, dès lors, de développer notre capacité à reconnaître les traces du sacré même lorsqu'il ne se présente pas sous un aspect habituel à nos yeux, et à retrouver sa présence en divers lieux du territoire ${ }^{1}$. On pense tout d'abord, spontanément, aux sites caractérisés par la présence d'eaux, de sources ou lacustres, qu'elles soient bienfaisantes ou nocives. Les cas moins évidents ne manquent toutefois pas, comme le montre le fascinant exemple du complexe de Saint-Martin-de-Corléans, à la périphérie d'Aoste, avec sa superposition millénaire de structures, parfois très éphémères².

Concrètement, une première modalité cultuelle concerne les espaces définissables, de manière générale, comme domestiques et résidentiels : on a, par exemple, interprété en ce sens la fonction d'une structure située dans la cour d'un grand édifice du centre peucète de Torre di Castiglione (commune de Conversano) contenant de la vaisselle céramique d'époque archaïque tardive et des restes d'animaux brûlés (MASTRONUZZI, 2005, p. 51 et suiv.). De manière analogue, avant même les découvertes de Torre di Satriano, on a émis l'hypothèse selon laquelle certaines constructions de type résidentiel, ou caractérisées par des structures planimétriques semblables, et construites au cours du v viècle à Lavello, en Daunie ofantine, auraient pu accueillir des cérémonies dont la nature ne se limitait pas à la convivialité, en raison de la présence, dans l'une d'elles, d'une fosse qui en contenait les restes (MUTINO, 2012 ; BOTTINI, MARCHI, 2016, p. 12). Plus récemment, le réexamen d'un complexe résidentiel, qui a aussi pris pour objet le contenu de deux petits puits, a conduit à des considérations similaires pour Arpi (LEONE et al., 2015). Cette hypothèse a enfin été clairement confirmée par deux structures palatiales érigées à deux moments distincts du IV siècle, dans des régions plutôt éloignées l'une de l'autre : la première à Roccagloriosa, le grand centre de l'arrière-pays tyrrhénien dont la domination s'étendait sur la partie la plus occidentale de la Lucanie, fondé selon toute apparence sur l'agrégation de groupes aristocratiques; le second à Pomarico Vecchio, aux confins de la Peucétie, dans le 
contexte d'une urbanisation achevée. Dans ces deux structures, on a mis au jour un édicule : à Pomarico Vecchio, on a découvert, à proximité, un socle qui devait soutenir une statue (BARRA BAGNASCO, 1997, p. 16 et suiv.) ; à Roccagloriosa, il contenait des restes de sacrifices, des terres cuites et des céramiques pour la plupart de petites dimensions, selon un modèle cultuel de type chthonien qui, bien que sans doute voué à une entité assimilée à l'Héra des Achéens, témoigne de rites que les archéologues appellent des gentilicia sacra (GUALTIERI, FRACCHIA, 1990, p. 103).

On peut replacer dans cette perspective, à condition de dépasser des oppositions plus idéologiques qu'historiques et archéologiques, le cas très célèbre de l' " édifice V » du " Timpone della Motta " de Francavilla Marittima (identifiée à Lagaria, mais voir PAOLETTI, 2014). Les archéologues hollandais y ont reconnu, dans ses phases initiales, un lieu de culte, et ce, pour reprendre les termes de Pier Giovanni Guzzo, en reconstruisant une «idéologie religieuse œenôtre de l'âge du fer, constituée d'éléments analogues à ceux de l'idéologie religieuse grecque, qui se manifeste à travers des preuves matérielles (GUZZO, 2011, p. 222 et suiv. ; sur le contexte archéologique, voir désormais PACCIARELLI, 2016). Remarquons au passage que cette position présente plusieurs points communs avec celle de Felice Gino Lo Porto et Francesco Ranaldi à propos de Braida di Vaglio, bien qu'elle se fonde sur une thèse opposée : " au moment de la colonisation, les "Italiques" avaient atteint un niveau de civilisation égal à celui des "Grecs" " (KLEIBRINK MAASKANT, 2003, p. 8). Toutefois, dans le même contexte, l'auteure exprime un point de vue moins rigide sur cette question spécifique, lorsqu'elle écrit que " la "Casa delle tessitrici", sur l'acropole, revêtait selon toute probabilité la même double fonction que celle que l'on attribue à l'architecture des demeures des souverains du premier âge du fer : ces édifices sont en effet représentatifs du prestige de l'aristocratie dominante, mais il s'agissait aussi d'édifices de culte " (KLEIBRINK MAASKANT, 2003, p. 62).

Par ailleurs, et compte tenu précisément de la centralité reconnue des groupes de parentèles, le culte des ancêtres - réels ou mythiques - origine et garantie aussi bien de l'identité même du groupe (comme l'a souligné à juste titre OSANNA, 2008, p. 149 et suiv.) que de la légitimité de son rôle social, voire de la transmission de son pouvoir de génération en génération, a occupé une très grande place. Cet aspect, commun à d'autres cultures européennes (FERNÁNDEZ-GÖTZ, 2016), concerne le vaste domaine de l'idéologie funéraire prise au sens strict, mais il peut aussi avoir des effets sur l'aménagement même des structures résidentielles, comme le prouvent les découvertes effectuées sur l'acropole de Monte Sannace, ou encore favoriser l'apparition d'aménagements particuliers faisant penser à une éventuelle implication de la communauté, au-delà du groupe de la parentèle ; ce fut par exemple le cas en Daunie (sur ces deux cas, voir infra).

Enfin, il convient de faire au moins une allusion rapide à la fréquentation possible, par des indigènes, de sanctuaires édifiés en des lieux placés sous domination grecque. Dans le cas du sacellum mis au jour à Imbelli, près de Campora San Giovanni, dont on a imaginé qu'il se situait sur un territoire dépendant de la ville de Temesa, la découverte d'une série de fibules à arc revêtu, lors des fouilles, a été interprétée comme un indice de la consécration d'un vêtement féminin de type local (LA TORRE, 1996, p. 716). Cependant, rien ne permet d'établir l'identité de la personne à l'origine de cette consécration, ni de reconstituer les modalités de la cérémonie ${ }^{3}$. Du reste, toujours au sanctuaire d'Imbelli, un empilement d'au moins 73 lances, associées à un objet dans lequel on a reconnu un sceptre, a été considérée de manière convaincante comme " une grande offrande publique, un véritable trophée, donc, composé d'armes soustraites à l'ennemi lors d'une bataille et offertes toutes ensemble à la divinité ", l'ennemi, en l'occurrence, étant italique (LA TORRE, 2011, p. 74). 


\section{Formes et structures de la religiosité collective}

Dans les pages qui suivent, on tentera de dresser le bilan de nos connaissances relatives à des lieux et des structures où l'on a pu discerner les traces d'actes cultuels concernant la communauté (et ceux qui, bien que n'y appartenant pas, la fréquentaient), sur les territoires actuels des Pouilles, de la Basilicate et de la Calabre septentrionale. On adoptera une forme synthétique, lorsqu'il existe des synthèses solides, mais on s'efforcera en tout état de cause de rendre compte des données scientifiques et des apports critiques les plus récents ${ }^{4}$.

\section{La Messapie}

La situation de loin la mieux connue est sans aucun doute celle de la péninsule du Salento. L'adoption relativement précoce de l'écriture, survenue au cours du vi siècle avec la création d'un alphabet "dérivé pour l'essentiel de l'alphabet laconien de Tarente ", mais qui dénote aussi d'autres contacts (MARCHESINI, 2013), apparaît ici fondamentale. Elle résulte d'une interaction culturelle aux origines très anciennes qui se conjugue, selon des modalités que nous ignorons pour une large part, à des relations "politiques " qui aboutirent parfois à des conflits militaires d'une extrême dureté, comme nous le rappellent, d'une part, Hérodote (VII, 170) et, de l'autre, les deux très célèbres et très discutées offrandes votives de Tarente 5 .

Grâce aux épigraphes, nous connaissons une série de théonymes qui sont parfois manifestement calqués sur des divinités grecques, en particulier Zeus, Déméter, Aphrodite (DE SimOnE, 1988 ; POCCETTI, 2008b), auxquels il faut associer les résultats de nombreuses investigations menées sur le terrain (MASTRONUZZI, 2005, p. 133 et suiv.). Par ailleurs, il ne faut pas négliger le fait que l'apposition d'inscriptions devient en elle-même, à compter de la seconde moitié du IV siècle, l'acte principal du culte, comme le démontrent les 400 mètres carrés de graffitis découverts sur les parois de la Grotta della Poesia de Roccavecchia. Parmi la quarantaine d'inscriptions, certaines, dans lesquelles apparaît le mot tabara, traduit en général par "prêtresse ", se réfèrent à Déméter et Aphrodite. Le récent examen approfondi des rares inscriptions dont on connaît le contexte archéologique (funéraire) a conduit Edward Herring à conclure que ce qualificatif de tabara " constituait une composante essentielle de l'identité sociale de la défunte. Il était important, aux yeux de ceux qui procédaient à l'enterrement d'un individu, de consigner ce statut, soit pour leur propre bénéfice, soit pour celui de la défunte, soit encore pour celui de la divinité que la tabara avait servie " (HERRING, 2007, p. 144). On peut dès lors se demander si la meilleure traduction de ce mot ne serait pas plutôt " adepte ", qui reste plus neutre quant à l'éventuelle fonction publique de la personne désignée, impliqué en revanche par le terme de "prêtresse ». L'intense activité archéologique de ces dernières décennies a permis de reconstituer le " contexte matériel » au sein duquel se tenaient les pratiques cultuelles et les dispositifs concernés : située face à la Grotta Porcinara, l'eschara de Santa Maria di Leuca est utilisée, entre la fin du VIII et le milieu du $\mathrm{VI}^{\mathrm{e}}$ siècle, aussi bien par des autochtones que par des navigateurs grecs. On a ainsi mis en évidence le rôle central joué par les grottes et les cavités (sur Santa Maria di Agnano, voir en dernier lieu Coppola et al., 2008), encore fréquentées même après la fin de l'autonomie politico-culturelle des Messapiens et parfois accompagnées, dans les espaces environnants, d'aménagements servant au déroulement de cérémonies collectives (c'est par exemple le cas du site de Monte Papalucio, près d'Oria : MASTRONUZZI, 2005, p. 83 et suiv. ; MASTRONUZZI, 2013).

D'autre part, les études les plus récentes (D'ANDRIA, MASTRONUZZI, 2008) ont mis en lumière un autre type d'installation cultuelle, centré cette fois sur des cippes, parfois agrémentés d'éléments décoratifs empruntés au répertoire de l'architecture monumentale grecque et adaptés à telle ou telle situation spécifique (dans un rapport qui fait penser à celui 


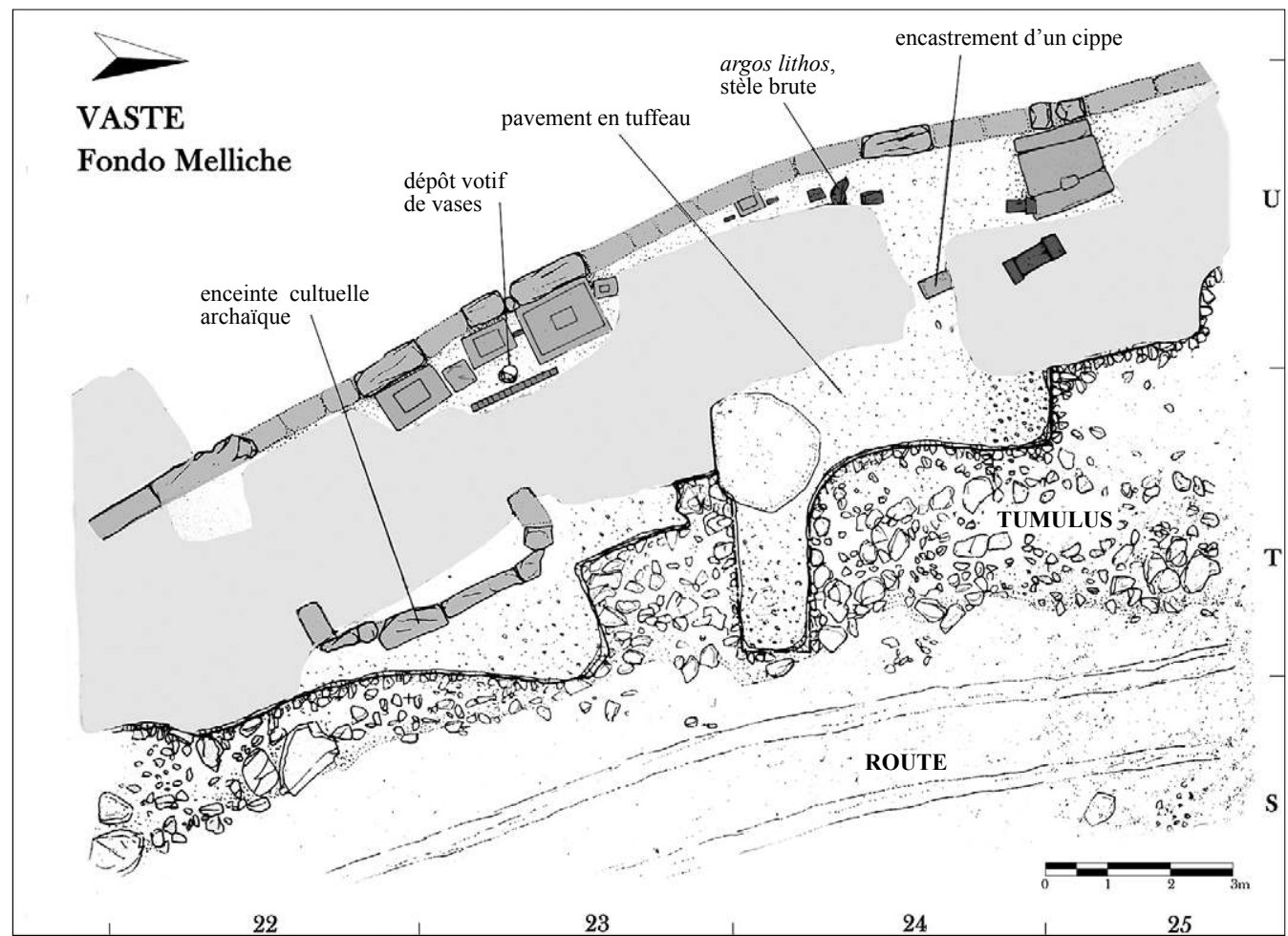

2. Site de Fondo Melliche, époque archaïque, Vaste (Poggiardo, Lecce), extrait de MASTRONUZZI, 2005.

évoqué plus haut à propos de l'alphabet local). Selon toute apparence, ils furent adoptés non seulement dans le cas de dispositifs que l'on pourrait définir comme des "sanctuaires des murailles " (ou, peut-être mieux encore, "des portes "), par exemple à Cavallino et Vaste, mais aussi dans de véritables lieux de culte, comme l'a montré la découverte de celui, exceptionnellement bien conservé, du Fondo Melliche, toujours à Vaste ${ }^{6}$ (fig. 2 ; MASTRONUZZI, 2005, p. 126 et suiv., fig. 41).

Cette découverte a également permis de mieux comprendre celles, antérieures, du site côtier de Scala di Furno (commune de Porto Cesareo) et de proposer une reconstitution, sous des formes analogues, du contexte d'où provenait la célèbre statue en bronze de Zeus mise au jour à Ugento. D'un point de vue plus strictement religieux, son lien apparaît renforcé avec l'entité divine connue sous le nom de Zis Batas, pendant masculin des cultes à caractère chthonien liés à Déméter et Korè (voir aussi HINZ, 1998, p. 196 et suiv.) présents aussi, comme on le verra plus loin, sur d'autres territoires.

L'un des aspects les plus intéressants du sanctuaire de Monte Papalucio est la présence d'un groupe de céramiques à figures noires spécifiquement destinées au culte, pour la plupart des hydries dans lesquels on a proposé de voir la production d'un « artisan immigré, d'origine gréco-orientale ou étrusque, suivi ensuite par des élèves d'extraction indigène " (MONTANARO, 2010, p. 248). Il s'agit là, en tout état de cause, d'une preuve supplémentaire du rôle central joué par ce type de dispositifs dans la vie des communautés messapiennes.

\section{La Peucétie}

Le " modèle " messapien ne semble pas s'être étendu à la partie centrale de la région, à savoir celle qui comprend le territoire de Matera situé autour du fleuve Bradano, aujourd'hui en Basilicate (BOTTINI, MARCHI, 2016, p. 20 et suiv.). 
On a déjà fait allusion au rôle très significatif du lien aux ancêtres, tout du moins dans le cas des élites. C'est en effet ce qui se dégage clairement des recherches menées sur l'acropole de Monte Sannace : une synthèse récente (GALEANDRO, PALMENTOLA, 2013) a ainsi confirmé, en premier lieu, la disposition de plusieurs tombes datant de la même période sous le pavement d'une structure d'époque archaïque, dont on avait déjà supposé par le passé qu'elle remplissait une fonction cultuelle. Elle a fait réapparaître, en second lieu, une relation analogue avec les ancêtres, s'exprimant sous des formes diverses, durant la seconde moitié du IV siècle, époque à laquelle semble remonter aussi la délimitation, par un mur, d'une aire sacrée privée de cette connotation spécifique.

Abstraction faite de la présence possible de lieux de culte à l'échelle locale, dont témoigne pour l'essentiel la découverte d'objets interprétables comme des offrandes, le seul véritable sanctuaire reconnaissable en tant que tel semble être celui de Lamia San Francesco, sur les hauteurs des collines, là où s'organisa le centre important et ancien de Timmari, dans la partie la plus interne du " canton " peucète. Aujourd'hui en Basilicate, ce lieu est de ce fait généralement qualifié de " lucanien " (voir par exemple HINZ, 1998, p. 201 et suiv. : " das östliche Lukanien "), bien qu'il soit difficile de rattacher le sanctuaire à ce type de contexte historique et culturel et qu'il apparaisse au contraire aisé de reconnaître, aussi bien dans son érection que dans l'identification des deux divinités concernées, un contact avec les poleis de la côte ; on observera d'ailleurs un phénomène analogue, plus loin, à propos de Garaguso. Comme on l'a déjà montré par le passé (LO PORTO, 1991, p. 62 et suiv.), les fouilles n'y avaient pas été menées de manière irréprochable, entre la fin du XIXe siècle et l'année 1929, dans deux zones distinctes (A et B), séparées par une distance d'environ 220 mètres. Selon toute apparence dotées chacune de sacella, du moins à en juger par les informations relatives à la mise au jour de blocs et de deux antéfixes (qui renvoient en tout état de cause à une phase récente), elles se distinguent par la présence éventuelle d'un mur de temenos sur la seconde (B). La première $(\mathrm{A})$ était reliée à une source. Un réexamen analytique récent des matériaux votifs, catégorie par catégorie (plusieurs contributions à ce propos dans BATTILORO, OSANNA, 2011 , p. 58 et suiv.), a permis de situer les débuts de la fréquentation du sanctuaire au cours $\mathrm{du} \mathrm{VI}^{\mathrm{e}}$ siècle. Documentée aussi bien par la céramique matt-painted locale que par la céramique de fabrication gréco-coloniale, qui atteignit son apogée au IV siècle, avec la présence de tous les types d'offrandes caractéristiques de cette époque, elle déclina au siècle suivant. Un petit ensemble de skyphoi et de cratères skyphoïdes ornés de l'image d'une torche en forme de croix (ibid., p. 61, fig. 5) dont on peut supposer qu'elle remplissait une fonction cérémonielle, renvoie au culte de Déméter. L'invocation ПАI (pai) tracée au centre d'une coupe à vernis noir de la fin du IV siècle découverte dans la zone A (ibid., p. 119 et suiv., fig. 5), et que l'on s'accorde à rapporter à Korè, la " jeune fille ", par antonomase, renvoie au même culte (fig. 3a-b). On a par ailleurs mis au jour dans la zone $\mathrm{B}$ un fragment de cratère à figures rouges de la fin du IV siècle portant l'inscription Aphrodite (ibid., p. 101 ; LO PORTO, 1991, pl. LXXVI, nº 217), où l'on a reconnu l'indice d'une dévotion complémentaire. Enfin, autre élément distinctif par rapport à la Lucanie, rien n'évoque ici la déesse Mefitis, dont on parlera plus loin.

Les fidèles étaient aussi bien des femmes, comme l'indiquent quelques bijoux (ibid., p. 85), que des hommes, qui ont laissé sur place des armes, réelles ou miniatures, dont il sera à nouveau question plus loin (infra, p. 78).

\section{La Daunie}

La partie la plus septentrionale de la région où se développa la culture apulienne semble s'être distinguée du reste du territoire dans ses manifestations collectives de religiosité, plus encore que dans d'autres domaines : on n'y a découvert aucun aménagement comparable 

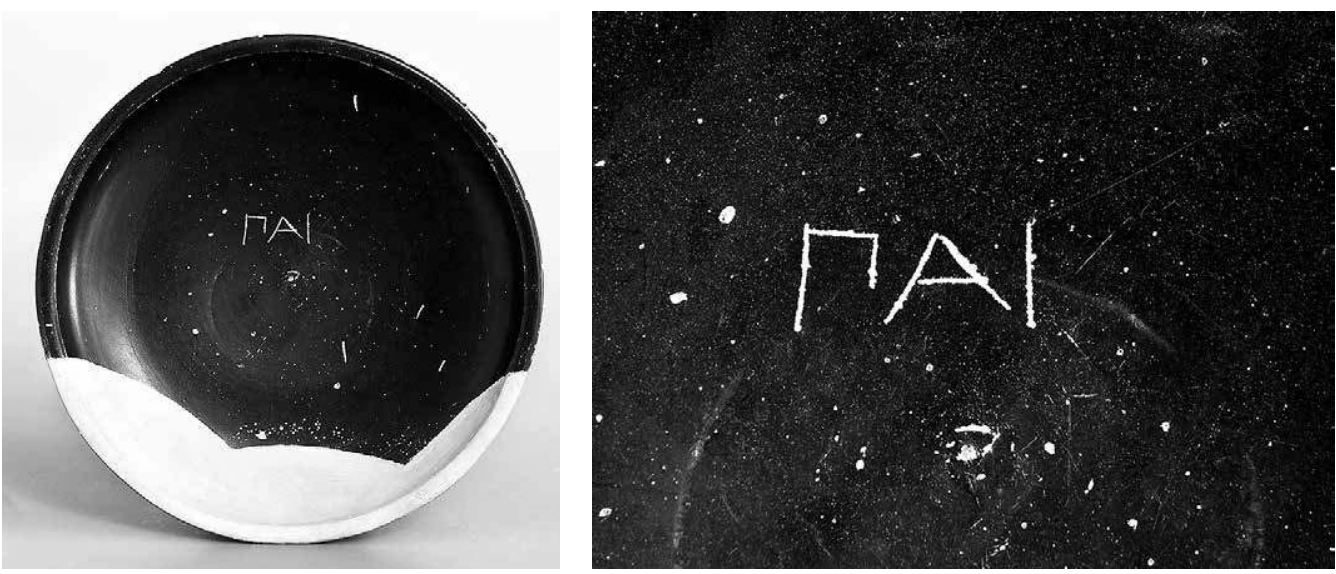

3a-b. Coupe à vernis noir, et détail de

I'inscription $\Pi A \mathrm{~A}$ (pai) [b], fin du IV siècle av. notre ère,

Timmari (Matera),

Matera, Museo

Archeologico Nazionale D. Ridola.

à ceux de la Messapie, ni la moindre trace d'une fréquentation majeure sur le long terme analogue à celles dont on vient de parler dans le cas de Timmari, et reconnaissable à travers des dépôts votifs.

Le cas bien connu du stipes du Belvedere de Lucera confirme bien cette observation : dans les conclusions de son édition critique, Maria Cecilia D'Ercole n'a en effet rattaché à un éventuel lieu de culte actif avant la précoce colonisation romaine de 315/314 avant notre ère qu'une série d'antéfixes (D'ERCOLE, 1990, p. 289). Il faut cependant garder à l'esprit qu'on connaît d'autres emplois, documentés, de cette catégorie particulière d'objets très répandue, qui n'est pas réservée à la couverture d'édifices. Le lieu de culte mis au jour dans la zone de Gravetta di Lavello semble également avoir été fréquenté à une époque successive (BOTTINI, TAGLIENTE, FRESA, 1991 ; BOTTINI, GUZZO, 1992).

En partant du réexamen des découvertes effectuées sur la Collina del Serpente d'Ascoli Satriano, où la convergence de structures et d'aménagements de différentes natures dans un même espace, compliquée, en outre, par la superposition de témoignages d'époques successives, est difficile à interpréter, Massimo Osanna a accompli une importante tentative de synthèse. Il y a établi des comparaisons et, là où c'était possible, des relations, entre les données relatives à ce centre de la plaine du Tavoliere, jusqu'alors plutôt sous-évalué, et celles concernant Lavello, un site tout aussi considérable occupant une position à peu près symétrique, sur le versant opposé du fleuve Ofanto (BOTTINI, MARCHI, 2016, p. 10 et suiv.). C'est ainsi que l'on a pu reconstituer la façon dont un édifice de l'époque archaïque tardive, destiné à centraliser toutes les fonctions, à l'instar de ceux de Lavello cités plus haut, a été remplacé par un édifice que caractérisent la « restructuration des couvertures et la redéfinition de l'espace extérieur ", où " la sacralité du lieu devient plus que jamais manifeste, comme l'atteste l'étonnante accumulation de matériaux découverts en des associations significatives aussi bien à l'intérieur [...] qu'à l'extérieur ". On a aussi mis au jour dans les environs (destinés à des cérémonies d'un autre type ?) deux " petites pièces de moins de 10 mètres carrés, dont il a surtout été possible de récupérer les couvertures en tuiles permettant de reconstituer un toit, selon toute probabilité à double versant, décoré d'antéfixes ; il ne reste en revanche aucune trace de l'élévation, à l'évidence construite avec des matériaux périssables, bois ou briques crues. On peut déduire la fonction de ces structures, au sein de l'aire sacrée, à partir des matériaux qu'elles contenaient au moment de l'effondrement des couvertures. Sous les tegulae et les imbrices, on a en effet découvert, au contact du plancher, un riche ensemble de céramiques appartenant à différentes classes [...] datables entre la toute fin du $\mathrm{IV}^{\mathrm{e}}$ et le tout début du III siècle. " Il ne s'agit d'ailleurs pas de phénomènes isolés : un autre 


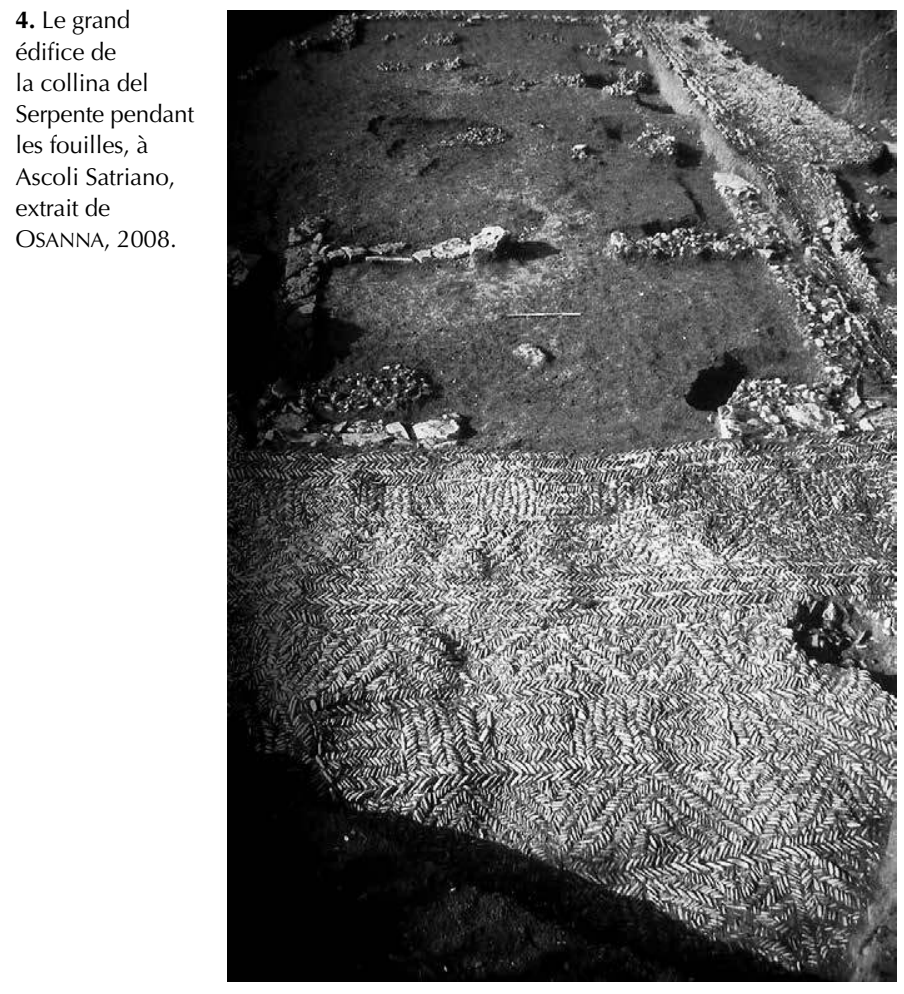

4. Le grand Serpente pendant les fouilles, à Ascoli Satriano, extrait de OSANNA, 2008.

essai mentionne ainsi " deux oikoi donnant sur une spectaculaire voie processionnelle, composée d'un pavement en mosaïques de galets à la syntaxe géométrique complexe, et contenant des objets analogues (OSANNA, 2008, p. 155 et suiv.).

Bien plus que les antéfixes, ces « pavements en mosaïques de galets " (fig. 4) constituent l'élément qui signale mieux qu'aucun autre, sur les sites de culture daunienne, les aires à fonction cérémonielle ; ils sont systématiquement associés à des sépultures monumentales et à de structures auxquelles on a successivement donné diverses interprétations, à Lavello même (contrada Casino, où l'on avait pensé à la présence de petites constructions périssables : BOTTINI, FRESA, TAGLIENTE, 1990, p. 246 ; voir désormais Mutino, 2012) et sur le territoire de Lucera (Masseria Casanova : CORRENTE et al., 2008, p. 382 et suiv.). Il est probable, en outre, que l'édifice récemment découvert à Troia soit à interpréter dans ce sens (DE SIENA, 2014 , p. 478 et suiv.). On trouve aussi ces " pavements " dans le petit édifice réapparu à Tiati (lieu-dit Regio Tratturo, sur le territoire de la commune de San Paolo di Civitate), où la présence de terres cuites figuratives de grandes dimensions semble confirmer qu'il s'agissait d'un véritable temple (MAZZEI, 2010, p. 182 et suiv.), dont il faudra toutefois préciser le développement chronologique, au cours du IV siècle. Il s'agit en tout état de cause d'un unicum, sans doute fruit de relations avec la Campanie voisine.

Il faut enfin accorder une mention tout à fait particulière à un ensemble architectural de Lavello, dont il apparaît aujourd'hui encore difficile de proposer une analyse exhaustive (TAGLIENTE, 1991 ; MASTRONUZZI, 2005, p. 65 et suiv., fig. 13). On y a découvert, sur un espace occupé en grande partie par des tombes de très haut niveau datées entre la fin du ve et le tout début du III e siècle, une enceinte rectangulaire (péribole alpha), dont seule la partie inférieure s'est conservée sur une portion de son extension. Orientée selon un axe Est-Ouest, d'une largeur d'environ 16 à 17 pieds et d'une longueur située entre un minimum de 23 à 24 pieds et un maximum de 33 à 34 pieds (soit environ 5 mètres sur au moins 7), elle fut érigée en scellant une série d'au moins six petits puits remplis de très nombreux fragments de vases de petites dimensions, renvoyant à un nombre très limité de formes, destinés aussi bien à verser du liquide qu'à en contenir, et tous caractérisés par d'abondantes traces de combustion. Un travail minutieux de restauration a permis de confirmer qu'ils avaient été déposés au même moment, et répartis de manière aléatoire entre les petits puits : des fragments d'un même vase ont en effet abouti dans des fosses différentes. De ces éléments, on tire l'impression que les puits conservaient des récipients, vraisemblablement datables du deuxième quart du IV siècle, utilisés lors d'une cérémonie prévoyant l'usage du feu, et par conséquent de nature sacrificielle, célébrée pour consacrer l'espace entouré par la suite d'une enceinte. Cet espace peut donc être interprété comme la transposition sur terre d'une partie de la voûte céleste, centré sur le parcours apparent du soleil, et désigné par le mot latin templum, « espace défini 
par le sacré ". Varron indique en effet à ce propos (De lingua latina, VII, 6) : "Templum se donne dans trois acceptions différentes, soit par rapport à la nature ou au ciel, soit par rapport aux auspices ou à la terre, soit par rapport aux enfers et par analogie ${ }^{7}$. "

Non loin de là, bâti selon la même orientation mais dans un axe différent, un petit édifice fermé (oikos gamma) était doté d'une lourde toiture et de parois enduites d'un mélange d'argile et de paille à décor estampé, dont les motifs reprennent pour la plupart des frises architecturales, mais qui pourraient renvoyer aussi à la céramique italiote de la même époque. Derrière cet édifice, on trouve une autre parcelle de "pavement de galets ", construite une fois de plus en relation avec des sépultures situées à proximité. Cet ensemble formé d'un oikos et d'un peribolos avait été précédé d'aménagements de nature précaire, peut-être en bois, qui en avaient préfiguré les formes, les dimensions et la disposition (oikos delta, peribolos beta). Cela nous permet de dater au plus tard du siècle précédent le développement de typologies de culte qui semble être l'apanage d'un unique noyau, celui-là même auquel il faut attribuer ces tombes 607 et 609, dont le mobilier funéraire comprenait un ensemble cohérent de petits vases appartenant à la même catégorie que ceux utilisés au cours de la cérémonie de consécration - ce qui n'exclut pas pour autant la possibilité d'une participation de la totalité de la communauté à cette cérémonie. Une hypothèse interprétative, déjà formulée par le passé, reconnaît dans cette enceinte le lieu destiné à l'observation du vol des oiseaux, ce qui supposerait l'existence d'une continuité rituelle avec le célèbre templum augurale républicain d'une ville voisine, Bantia (aujourd'hui Banzi ; voir en dernier lieu, en particulier pour le déchiffrement des sigles tracés sur les cippes, CARANDINI, 2006, p. 424 et suiv.). Après avoir été discutée par la critique (D'ALESSIO, 2013, p. 320), cette hypothèse a été renforcée par l'établissement de remarquables analogies structurelles et dimensionnelles avec un dispositif appartenant à un contexte non seulement plus ancien, mais en outre tout à fait différent et plutôt éloigné : celui des Paléovénètes d'Este (RUTA SERAFINI, SAINATI, 2002). Elle n'est d'ailleurs incompatible ni avec la vocation principalement funéraire de la zone (où l'on ne trouve en revanche pas le moindre vestige d'habitations), ni avec l'évidente analogie dimensionnelle entre l'oikos et la structure centrale du modèle de sanctuaire caractéristique de la Lucanie voisine (voir infra).

Pour compléter cette vue d'ensemble, il convient de rappeler la découverte d'un dépôt votif qu'il faut, selon toute apparence, mettre en relation avec des structures très sommaires, et qui a été mis au jour au lieu-dit Fontana dei Monaci, près de Banzi, à l'extrémité méridionale du territoire des Dauniens. Il a livré un important ensemble de modèles miniaturisés en métal, de fibules et surtout d'armes (MASSERIA, 1999 et 2000, p. 173).

\section{La Mésogée des Apennins à l'époque archaïque}

Malgré l'existence d'une exception remarquable et frappante, la partie la plus interne de l'actuelle Basilicate, relevant des deux cultures œenôtre et " nord-lucanienne " (BotTINI, MARCHI, 2016), constitue à n'en pas douter le territoire le plus avare en témoignages qui relèveraient de pratiques religieuses, abstraction faite de celles appartenant à la sphère funéraire. Dans un contexte où l'on ne dispose d'aucune trace d'aménagement collectif, on ne s'étonnera pas non plus de l'absence d'éléments permettant de soutenir que certains lieux de culte liés aux eaux, fréquentés à l'époque suivante (par exemple à Chiaromonte : MASSERIA, 2000, p. 136 et suiv. ; MASTRONUZZI, 2005, p. 49 et suiv.), l'aient déjà été précédemment. La prudence, à ce propos, d'Osanna, quant au sanctuaire lucanien de Torre di Satriano, le seul à avoir fait l'objet d'une analyse complète (fig. 5 ; OSANNA, SICA, 2005 , p. 59, note 62), est emblématique de celle de l'ensemble de la communauté scientifique. Il faudrait par conséquent vérifier à nouveau, à la lumière des immenses progrès acquis dans l'étude de leurs classifications, les chronologies des objets cités pour étayer des 

lucanien "type ", schéma de I'aménagement d'origine, IVe siècle av. notre ère, Torre di Satriano, extrait de OSANNA, SICA, 2005.
5. Le sanctuaire

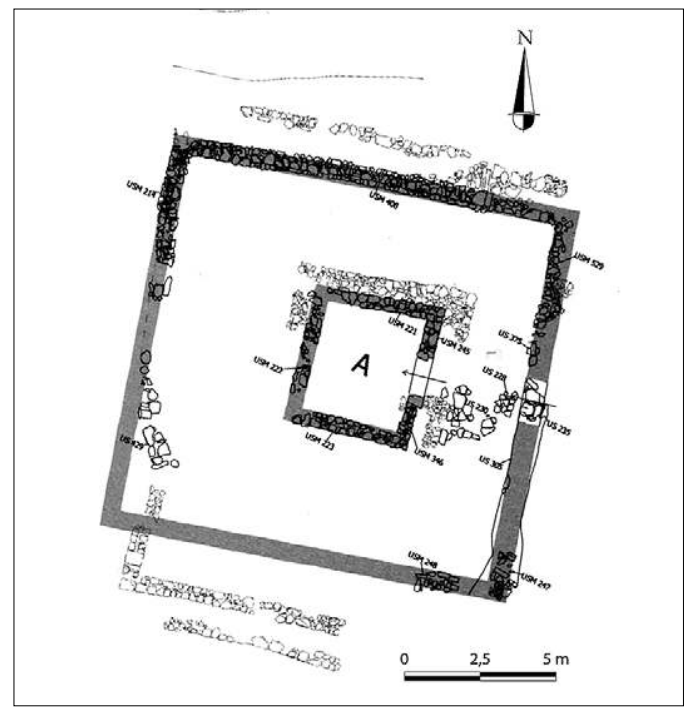

6. Modèle de temple contenant la statue d'une divinité assise, marbre, première moitié du Ve siècle av. notre ère, Garaguso, Potenza, Museo provinciale.

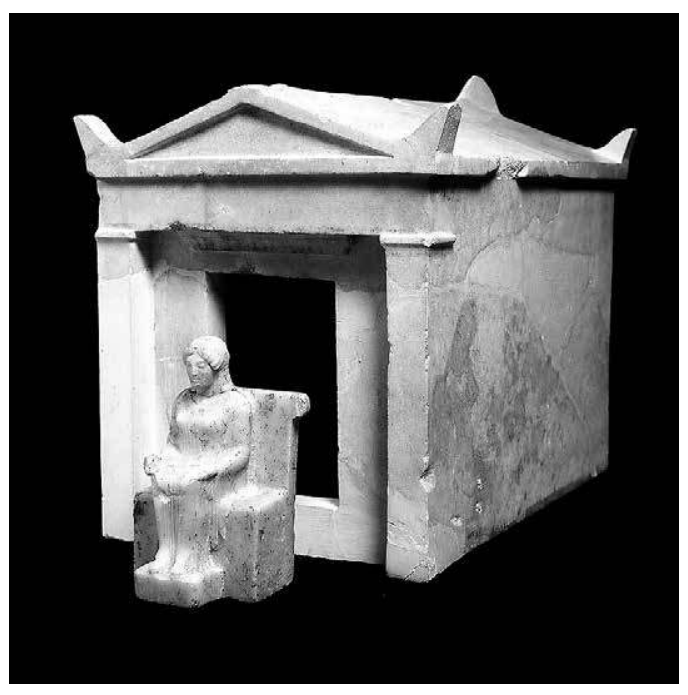

datations relevant de l'époque archaïque, par exemple dans le cas, toujours à Torre di Satriano, de l'édifice que des archéologues américains avaient défini jadis comme un Spring Sanctuary (HOLLOWAY, 1970, p. 32).

On peut cependant observer des éléments, dans l'arrière-pays de Métaponte, le long du torrent de Salandrella à sa source, puis du fleuve Cavone, qui se jette dans la mer à l'extrémité méridionale de la chôra de la polis, et qui constituent une exception : trois lieux de culte archaïques dont on peut, pour le moins, reconstituer l'existence. Le cas le plus célèbre, et de loin le plus intéressant, concerne Garaguso, site de culture œenôtre ; à la lumière d'études très récentes qui les ont littéralement rendues à la science, la présence d'au moins deux sanctuaires distincts, édifiés à peu de chose près au cours des mêmes décennies, durant la seconde moitié du VI ${ }^{\mathrm{e}}$ siècle, y apparaît dans toute son évidence. Le premier, au lieu-dit Filera, était assurément doté d'un véritable oikos, dont provient aussi le célèbre modèle de temple en marbre contenant la statue d'une divinité assise, constituant peut-être l'image cultuelle elle-même (fig. 6 ; MORET, 2014). Au second, au lieu-dit Grotte delle Fontanelle, il faut rapporter les dépôts, jusqu'ici considérés comme distincts, de Filiera et Fontanelle (BERTESAGO, GARAFFA, 2015). Il présente des caractéristiques tout à fait différentes : dénué d'aménagement, il ne nous est connu qu'à travers une série de dépôts effectués dans de petites anfractuosités et grottes naturelles ou artificielles, selon une modalité observée récemment à Entella, en Sicile, dans un lieu de culte identifié comme un thesmophorion (SPATAFORA, 2016, p. 7 et suiv.). Les offrandes en question apparaissent plutôt modestes ; et contrairement à ce que l'on a pu en revanche observer tout près de là, à Timmari, on est ici frappé par l'absence totale de céramique indigène. Et l'on ignore hélas la localisation actuelle d'un fragment de vase à figures noires sur lequel une femme tenant une fleur s'approche d'un autel où brûle un feu, analogue à ceux que l'on a découverts dans le sanctuaire de Monte Papalucio, à Oria (voir MONTANARO, 2010, p. 247, pour le développement de l'hypothèse d'une production coloniale). Dans l'ensemble, les ex-voto sont comparables à ceux qui ont été mis au jour dans les lieux de culte de la chôra; il semble donc impossible de déterminer s'ils y ont été laissés par des fidèles indigènes ou grecs.

Il s'agit là du seul exemple connu à ce jour de présence métapontine directe dans l'arrière-pays italique, comme le confirme une découverte effectuée au XIX ${ }^{e}$ siècle en aval de San Mauro Forte : le célèbre modèle en argile de tetragonos lithos portant une inscription dédicatoire à Héraclès, en dialecte achéen, de la part de Nikomachos kerameus, à l'évidence 
originaire de la ville voisine (BotTINI, LECCE, 2013). Placé sur l'itinéraire qui relie justement ces collines à la côte, ce site (lieu-dit Priati) devait donc servir de lieu de culte. Désormais de toute évidence perdu, les dynamiques spécifiques à ce lieu nous échappent. Il faut toutefois garder à l'esprit une donnée fournie par les analyses anthropométriques : la forte composante an-hellénique de la population de ce territoire, et ce déjà dès la période située entre le VII et le VI siècle (CARTER, 2006). Quant à la nature des objets choisis, les découvertes effectuées sur le site du sanctuaire de Fondo Melliche à Vaste, en Messapie, évoqué plus haut, étayent l'hypothèse selon laquelle elle ne correspondrait pas à un choix épisodique, mais au respect d'une forme spécifique de culte.

\section{L'aire tyrrhénienne du golfe de Sapri, entre la Basilicate et la Calabre}

On retrouve une situation d'interaction peut-être analogue par certains aspects sur la bande côtière tyrrhénienne : toujours dans un contexte œenôtre, on peut y retracer la présence de « communautés politiquement structurées (la touta), qui utilisaient l'alphabet achéen, et donc en étroit rapport avec Sybaris (sans négliger pour autant le rôle de Poseidonia) ", capables d'entretenir des relations " internationales " du type de celles que mentionne la très célèbre tablette en bronze provenant d'Olympie et relative aux Serdaioi ${ }^{8}$. Une confirmation définitive de cette hypothèse nous est d'ailleurs offerte par l'interprétation comme lex sacra (proposée par Emanuele Greco) de la désormais célèbre inscription archaïque tardive mise au jour à San Brancato di Tortora, rédigée dans une langue italique archaïque de même souche que l'osque (LAZZARINI, POCETTI, 2001; MASTRONUZZI, 2005, p. 118 et suiv.).

Contrairement à ce que l'on a constaté plus haut à propos de la partie la plus intérieure du territoire de l'actuelle Basilicate, il apparaît donc probable que le culte pratiqué non loin de là, dans l'arrière-pays de Maratea, à Colla-Serra la Città (commune de Rivello), remonte à la même époque (GALIOTO, 2011). On pourrait avancer une hypothèse analogue dans le cas de Nemoli, située à proximité (MASTRONUZZI, 2005, p. 81 et suiv.). Cette probabilité est cependant documentée uniquement par les productions artisanales et les pièces de monnaie contenues dans trois petits fossés, avec des restes organiques et des os brûlés, dont l'origine sacrale ne fait pas de doute, sans présenter pourtant de référence directe à la pratique cultuelle propre aux thesmophoria grecs. La structuration du sanctuaire eut donc lieu au cours du IV siècle, selon toute vraisemblance à travers la mise en œuvre du modèle décrit au paragraphe suivant, qui prévoit la construction d'un petit oikos quadrangulaire $(3,2 \times 3,2 \mathrm{~m})$ à l'intérieur d'une enceinte. On ne comprend pas bien, en revanche, la fonction d'une seconde construction. Il semble avoir été surtout fréquenté par des femmes, même si les vestiges d'armes (ceinturons et pointes d'armes longues) ne manquent pas. La mise au jour de statuettes en argile, représentant aussi bien des figures tenant des torches que des offrantes au porcelet, suggèrent une référence au culte de Déméter. Une situation analogue pourrait aussi être reconnue plus au Nord, à Palinuro (MASTRONUZZI, 2005, p. 88).

\section{La Lucanie au IV ${ }^{\mathrm{e}}$ siècle}

L'apparition de divers lieux de culte bien identifiables comme tels, caractérisés par une variété morphologique notable, constitue l'un des signes les plus manifestes de la réorganisation des sociétés que nous associons au nom d'ethnie "Lucaniens".

Celui de Fontana Bona, à Ruoti, qui ne présente en apparence aucune autre structure qu'un grand bassin, et que l'on ne connaît qu'à travers ses offrandes, relève de la typologie la plus simple. Comme à Torre di Satriano (OSANNA, SICA, 2005, p. 440), on compte parmi elles un grand nombre de thymiateria, qui ont fait penser à des " rituels visant à parfumer 
le lieu sacré au moyen d'arômes de diverses natures" (BARRA BAGNASCO, 2008, p. 203). Également centré sur les eaux, le complexe de San Pasquale, à Chiaromonte, était, quant à lui, formé d'une série de terrasses " traversées par un long parcours "cérémoniel", selon toute probabilité couvert et doté de portiques ", qui rappelle les aménagements dauniens (BIANCO, 2003, p. 75). Grâce à une révision attentive des données fournies par les fouilles, Olivier de Cazanove a néanmoins proposé une réinterprétation de l'ensemble de Serra Lustrante, à Armento. Traditionnellement considéré comme un sanctuaire, il y voit plutôt une structure "palatiale ", dotée d'un espace cultuel du type de ceux que l'on a déjà mentionnés plus haut (à Pomarico, à Roccagloriosa ; CAZANOVE, 201 l, p. 301 et suiv.). Il faut particulièrement souligner l'adoption de ce que l'on peut définir comme un schéma structurel (" un projet standard ", reconnu et analysé dans CAZANOVE, 2009). Au moins trois sanctuaires - le temple P de Civita di Tricarico, celui de Torre di Satriano, cité supra, et celui du lieu-dit Pila, à San Chirico Nuovo - sont en effet formés d'une pièce de dimensions modestes, à plan carré ou rectangulaire, inscrite dans une enceinte qui en répète la forme et destinée à accueillir les fidèles et leurs offrandes. L'espace central, définissable sur le modèle grec comme un oikos, était en revanche réservé à la divinité. La découverte, dans le cas de Pila, d'un fragment d'une statue féminine en argile d'une hauteur d'environ 2 pieds (fig. 7), qui constituait selon toute vraisemblance l'image cultuelle, le documente clairement.

Dans ce même lieu de culte, il est très significatif qu'une partie des offrandes votives soit constituée de petits modèles en argile de grands récipients à denrées alimentaires, révélant au moins un, et non le moindre, des desiderata de la communauté des fidèles ${ }^{9}$, formée à l'évidence de la multitudo agrestium citée par Tite-Live (VIII, 27) dans son récit des événements des années 325-324 avant notre ère. On ignore l'identité de la déesse. Comme dans le cas de Torre di Satriano (OSANNA, SICA, 2005, p. 423), on a discuté son identification avec Mefitis, la déesse " italique " par excellence, dédicataire, comme le prouve un corpus significatif d'inscriptions, du sanctuaire lucanien sans aucun doute le plus connu, monumentalisé à l'époque romaine, à savoir celui de Rossano di Vaglio, une localité voisine (FALASCA, 2002, p. 42 et suiv.). Les études les plus récentes sur ce site ont permis de mieux retracer le contexte des offrandes votives mises au jour lors de fouilles précédentes, et ont fait apparaître une donnée jusqu'alors restée en partie dans l'ombre : le dépôt, selon des modalités encore inconnues, d'armes offensives et défensives (casques, boucliers, jambières), mis hors d'état de fonctionnement par des déformations rituelles (BATTILORO, OSANNA, 2011, p. 221 et suiv.). Ces armes sont ici en nombre bien supérieur par rapport à ce que l'on sait d'autres lieux de culte lucaniens (Torre di Satriano, San Chirico Nuovo, Chiaromonte, Rivello) ou situés sur le territoire apulien limitrophe, par exemple Banzi et Timmari, où l'on a découvert uniquement, outre un ensemble de reproductions miniatures, des pointes d'armes longues, des flèches et quelques ceinturons. Un casque en forme de pileus (Armi, 1993, p. 211 et suiv.) a par ailleurs été découvert au lieu-dit Caporre (commune de Ferrandina), à peu de distance du territoire métapontin (MASSERIA, 2000, p. 65 et suiv. ; MASTRONUZZI, 2005, p. 54 et suiv., avec des imprécisions et une datation " ancienne " probablement erronée). À Rossano di Vaglio, la présence de plusieurs épées de type archaïque, et donc antérieures à la date de fondation du sanctuaire communément acceptée (milieu du IVe siècle), auquel il faut ajouter un magnifique épisème de bouclier en bronze jusqu'ici à peu près inédit (Le sacre acque..., 2003, p. 95), est particulièrement étonnante. Tous ces éléments pourraient révéler une forte connotation belliqueuse du sanctuaire à ses débuts, tout à fait en accord avec l'image des Lucaniens, celle d'une horde militaire, que nous fournissent aussi bien les premiers éléments de mobilier funéraire de Gaudo, à Paestum (CIPRIANI, 1996), que les peintures funéraires. En revanche, cette connotation est absente des mobiliers funéraires de la même époque mis au jour dans l'intérieur des terres de la Basilicate. 


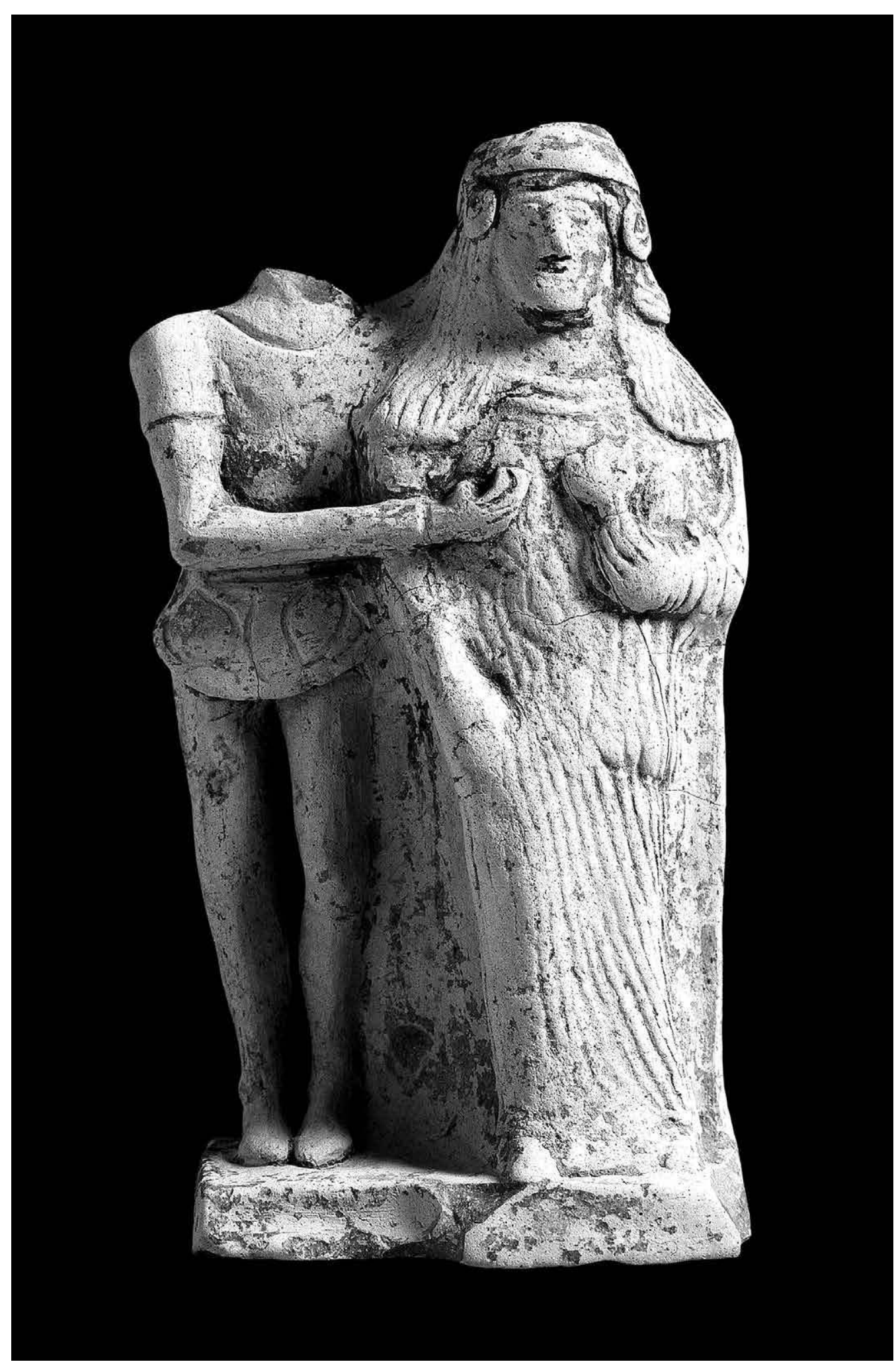

7. Statue féminine, argile, seconde moitié du IVe siècle av. notre ère, San Chirico Nuovo

(Pila, Potenza),

Potenza, Museo Archeologico Nazionale, inv. 98552. 
On peut ajouter qu'une orientation analogue se dégage de certaines des offrandes votives les plus anciennes, en argile ou en bronze, découvertes en dehors des limites territoriales de la Lucanie, dans ce qui fut très probablement le lieu de culte originel de la déesse Mefitis (POCCETTI, 2008b) : les eaux non seulement insalubres, mais rendues létales par des émissions d'anhydride carbonique et d'acide sulfurique, du petit lac de la Valle d'Ansanto (commune de Rocca San Felice), en haute Irpinie. Ce lieu sinistre était d'ailleurs bien connu des hommes de l'Antiquité, et ce n'est pas un hasard si Virgile le mentionne encore comme l'une des portes des Enfers (Énéide, VII, 563 et suiv.). Une étude très récente (Raimon Graells i Fabregat) consacrée à un trophée italique d'origine inconnue, et conservé à Munich, laisse entrevoir la possibilité qu'au moins le plus important des xoana retrouvées là ait rempli la même fonction (BotTINI, RAININI, COLAZzo, 1976, p. 376, fig. 6, nº 11). De manière plus générale, l'hypothèse d'une continuité avec les pratiques archaïques d'origine grecque, largement documentée en Occident, apparaît tout à fait probable (voir la récente synthèse de LA TORRE, 201 1, et un recueil de textes dans IANNELLI, SABBIONE, 2014; pour la Sicile : SPATAFORA, 2006).

\section{La religiosité individuelle et l'idéologie funéraire}

On a déjà fait allusion au caractère central que revêtait la relation avec les défunts dans la mentalité d'une large partie des peuples italiques, qui semblent très étrangers à la conception des morts enracinée aussi bien dans le monde grec que dans le monde romain : celle d'une présence contaminatrice qu'il fallait reléguer en dehors de l'espace réservé aux vivants. Dans les habitats de la plupart des entités cantonales indigènes, bien au contraire, les tombes ont constitué une présence constante, répandue un peu partout. Elles étaient tantôt insérées dans des dispositifs particuliers, comme dans la Daunie du IV siècle, signalées par des couvertures en tumulus ou encore formées de grands blocs de pierre, et parfois de semata; ce fut peut-être la fonction des "stèles dauniennes ", dont aucune, à ce jour, n'a cependant été retrouvée in situ. À Banzi, il est à peu près certain qu'on a employé, dans le même but, des antéfixes isolées - preuve supplémentaire que la présence de terres cuites architecturales n'implique pas nécessairement l'existence d'un édifice (MASTRONUZZI, 2011, p. 150).

Il s'agit presque exclusivement d'inhumations, sur le dos ou en position contractée, selon les appartenances cantonales ; l'incinération fut quant à elle adoptée dans un nombre très limité de cas, avec l'éventuelle exception, énigmatique, d'Irsina, dans la région du Bradanico (BotTINI, MARCHI, 2016, p. 23 et suiv.). Tout cela laisse supposer que le temps qui s'écoulait entre le décès et le dépôt du cadavre dans la tombe était occupé par des rituels. Bien qu'ils nous échappent totalement, on peut toutefois supposer qu'ils laissaient une certaine place à des actes de culte envers le défunt, ayant généralement pour objet son salut, et conséquences aussi, à partir d'une certaine époque, de l'adhésion à des formes particulières de religiosité. Il devait en résulter l'utilisation d'objets spécifiques, destinés à être intégrés aux mobiliers funéraires déposés aux côtés du mort. Les fragments de vases en argile crue désormais informes, mais qui conservaient malgré tout des traces de couleur, découverts à côté de céramiques de type ordinaire dans une importante tombe de la première moitié du IV siècle, à Lavello, nous offre un témoignage précieux de ce phénomène (lieu-dit Casino, tombe 745 : BOTTINI, FRESA, TAGLIENTE, 1990, p. 245 ; sur le trousseau funéraire : BotTINI, MARCHI, 2016, p. 16, 49). Sur le même site, plusieurs éléments témoignent par ailleurs du déroulement de cérémonies postérieures à la fermeture de deux tombes à chambre : des morceaux de chair ont en effet été déposés respectivement devant la porte de la tombe $\mathrm{n}^{\circ} 669$ et dans un petit puits situé à l'entrée du dromos de la tombe n ${ }^{\circ} 677$ (BOTTINI, FRESA, TAGLIENTE, 1990, p. 241, où la seconde 
porte erronément le $\mathrm{n}^{\circ}$ 667). Étant donné que dans le premier cas, en outre, un chien a été tué ${ }^{10}$, il semble évident que nous nous trouvons ici en présence des traces d'un véritable sacrifice, et non pas des restes d'un repas funéraire.

Concernant les objets eux-mêmes, Guzzo a récemment émis de sérieux doutes à propos de l'interprétation selon un point de vue religieux " cosmique " d'une cruche désormais célèbre, sans doute de production locale, déposée dans une tombe datable du $\mathrm{V}^{\mathrm{e}}$ siècle, à Ripacandida, un site de culture " nordlucanienne " de la région de Vulture : elle est ornée d'une figure humaine peinte, très schématique, surmontant un cercle contenant un foudre et entouré par sept étoiles. Il s'agit en tout état de cause d'un unicum absolu, au sein d'un contexte global peu enclin à la figuration, du moins pour ce qui concerne les céramiques. Il est en revanche difficile de nier l'appartenance à une catégorie d'objets dénotant clairement une fonction rituelle d'une singulière coupe en terre cuite découverte elle aussi à Lavello, dans une tombe masculine datable vers le milieu du VI ${ }^{\mathrm{e}}$ siècle, et dont le mobilier funéraire inclut, de manière très inhabituelle, un casque corinthien en bronze, une épée et des lances. Elle est comparable, d'un point de vue formel, à de nombreux
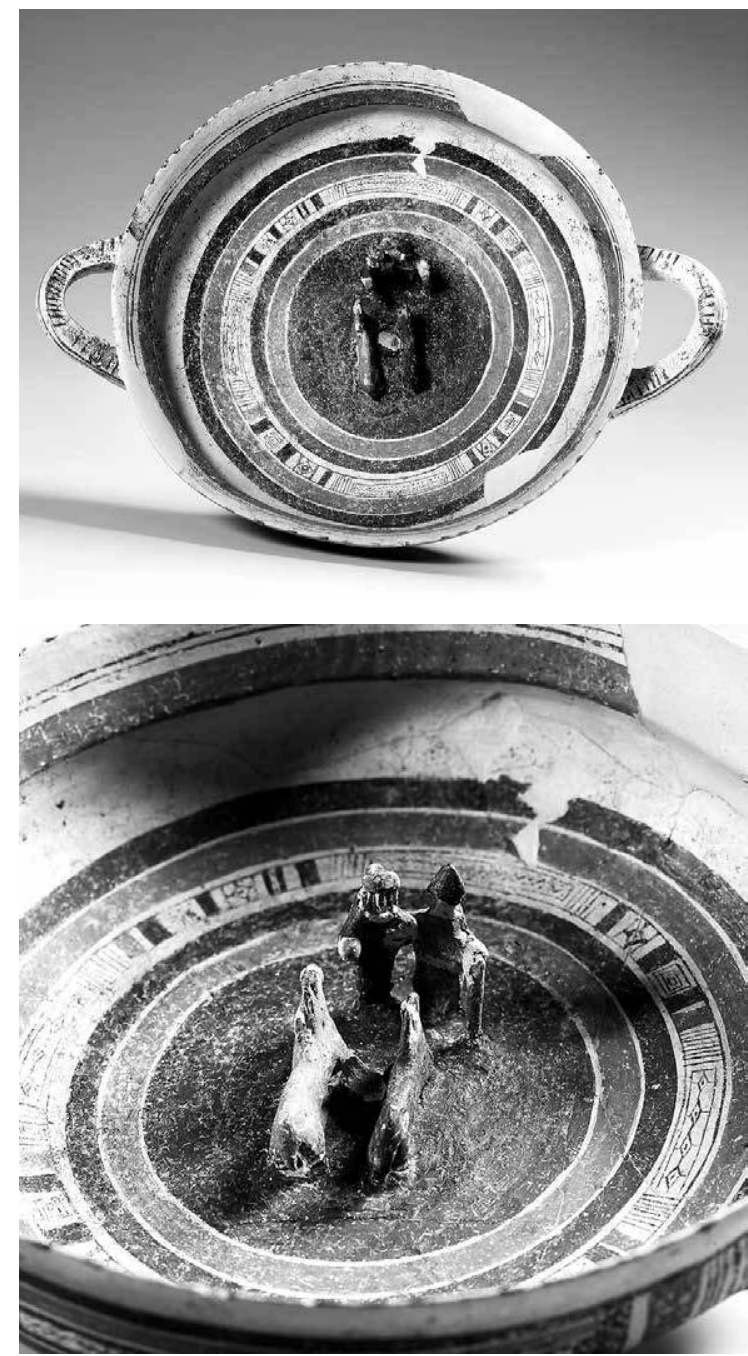

8a-b. Coupe peinte, et détail du groupe central $[b]$, argile, milieu du $\mathrm{Vl}^{\mathrm{e}}$ siècle av. notre ère, Lavello (tombe 796), Melfi, Museo Archeologico Nazionale del Melfese. autres exemplaires de production daunienne d'époque archaïque, provenant très probablement de Canosa. Le centre du récipient est toutefois occupé par un petit groupe modelé qui, de toute évidence, en contrarie la fonctionnalité : un couple composé d'un personnage masculin et d'un personnage féminin (TAGLIENTE, 1985/1986), vers lequel s'avancent deux chevaux, séparés à l'origine par un élément désormais disparu, peut-être un petit arbre (fig. 8a-b). Cet objet est intéressant à plusieurs titres. Les chercheurs ont d'emblée souligné la continuité de ce groupe par rapport à la tradition des symplegmata du premier âge du fer ${ }^{11}$, qui cependant, au moins dans le cas présent, ne se réfère pas à la sphère de la fertilité féminine, comme on l'a proposé pour le sanctuaire de Francavilla Marittima (KLEIBRINK, WeISTRA, 2013, p. 35 et suiv.). D'autre part, il faut souligner la façon dont la scène, replacée dans son contexte historique, rappelle l'importance de l'élevage équestre pour l'économie de la Daunie, un territoire que Strabon jugeait encore " excellent pour les troupeaux et les chevaux" (VI, 3,9=C284). Du reste, toujours à Lavello, une véritable sépulture a été réservée à l'un de ces animaux, selon une pratique assez courante parmi les populations italiques (BOTTINI, FRESA, TAGLIENTE, 1990, p. 238). Il convient par ailleurs de s'attarder sur deux détails de l'habillement des figures, dont 
les visages présentent, conformément à une tradition très répandue dans toute la Péninsule, des traits aniconiques. La femme porte une robe noire qui ne peut manquer de rappeler les scholies relatives à un passage de l'Alexandra de Lycophron, où il est justement dit que, dans des situations particulières, les Dauniennes melainan estheta phorousi ( " portent un habit noir " ; vers 1131, 1133, 1137, sur les implications de ce texte, voir HERRING, 2007, p. 138 et suiv. ; BOTTINI, MARCHI, 2016, p. 27). L'homme est, quant à lui, coiffé d'un couvre-chef en forme de cône comparable à celui apparaissant sur les "stèles dauniennes " mentionnées précédemment, et qui semble préfigurer celui que les peintres de vases italiotes attribuèrent plus tard aux guerriers italiques (RosCINO, 2009).

L'originalité de ce vase met ainsi en évidence sa nature non-fonctionnelle ; on peut toutefois se demander si d'autres vases dauniens, décorés de motifs figuratifs, n'auraient pas été fabriqués et ornés dans une perspective analogue. On pense par exemple à l'extraordinaire bassin de la fin du IV siècle, objet généralement associé fonctionnellement à une cruche, hélas arraché à son contexte de fouilles et conservé hors d'Italie (The Art of Italic Peoples..., 1993, p. 328 et suiv., $n^{\circ} 215$, où il est défini à tort comme un kalathos). Il rappelle, d'une part, l'askos Catarinella, à peine plus récent et décoré, comme on le sait, d'une scène de prothesis (sur son contexte historique et culturel, voir en dernier lieu DI FAZIO, 2009) ; il renvoie, d'autre part, à la découverte, déjà évoquée, dans certains lieux de culte, de vases ornés de représentations renvoyant à la sphère du sacré.

En se fixant l'objectif d'identifier les traces matérielles de comportements et de choix religieux apparus avec une préoccupation relative au destin des individus après leur mort, on explore un champ d'investigation aussi fascinant que semé d'embûches, où l'on court toujours le risque de la surinterprétation. D’ordinaire associée au désir de pouvoir bénéficier d'une protection surhumaine, cette préoccupation se situe au fondement du succès de formes de religiosité salvatrice, complexes et parfois insaisissables, dont Orphée est le symbole, sans en représenter pour autant le seul protagoniste.

La critique s'accorde à considérer que l'on doit à cet ensemble d'expectatives la prédilection, en premier lieu chez les Grecs, pour la représentation d'épisodes mythologiques ayant pour objet le rapt d'un mortel, ainsi transféré dans une dimension supraterrestre, par une divinité. Car ces images se prêtent bien à être "lues ", y compris par ceux qui ne possèderaient pas une connaissance approfondie du mythe traité, comme une claire métaphore de la protection divine accordée (aussi) après la mort. Nous reviendrons plus loin sur ce problème du niveau de communication linguistique et de transmission de la pensée. Et ce n'est sans doute pas un hasard si de telles images constituaient l'un des sujets favoris du peintre dit de Pisticci, premier peintre de vases à figures rouges de type attique actif à Métaponte à partir du milieu du ve siècle. Comme le laisse entendre le nom conventionnel qu'on lui a attribué et qui renvoie au principal site indigène dans l'arrière-pays de la polis grecque, sa production s'est pour l'essentiel retrouvée dans les demeures, puis dans les tombes, d'Italiques appartenant à diverses ethnies. Pour mieux replacer tous ces phénomènes dans leur contexte, il faut rappeler que, précisément pendant cette période, les pythagoriciens revenus à Métaponte, où ils occupèrent une position politique forte, semblent avoir adopté une attitude " inclusive " envers les non-Grecs. Si l'on en croit Alfonso Mele (MELE, 1981), cette attitude entraîna l'insertion, signalée par les sources antiques, de basileis et dynastai ( " rois " et " seigneurs ") italiques parmi les disciples de Pythagore, et conduisit même, plus tard, à l'ascension d'Italiques à la tête de cette école philosophique, à commencer par Aresas, "lucanien " (MELE, 2000, p. 313). Du reste, c'est au cours de ces années-là que fut édifié, au cœur de la cité achéenne, le petit ensemble architectural sacral où l'on a reconnu le manteion d'Aristeas, un personnage mythique étroitement lié à la figure de Pythagore et à un aspect tout à fait particulier du culte d'Apollon (DE SIENA, 1998, p. 166 et suiv.). 

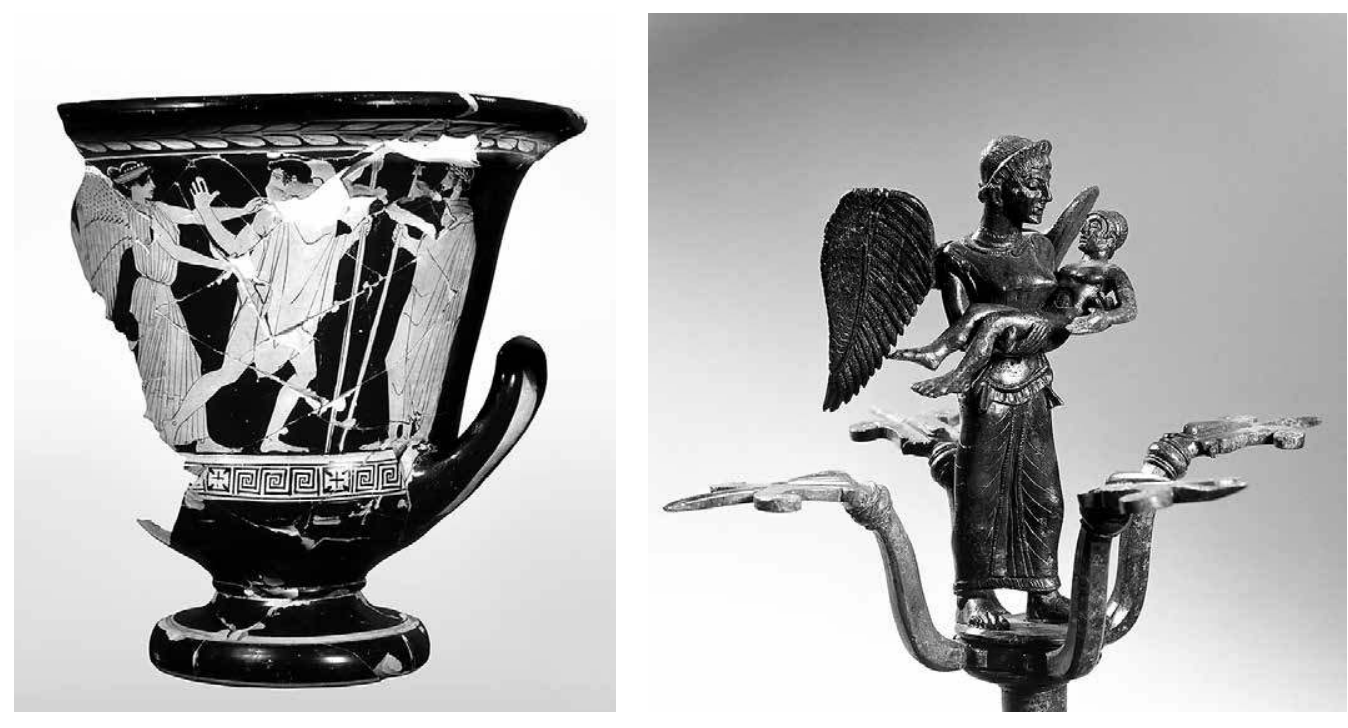

Il est peut-être légitime de rattacher à ces circonstances historiques particulières le cas de deux grandes tombes, assurément celles de personnages éminents du centre "nord-lucanien " de Ruvo del Monte. On a déposé dans la première, qui contient la dépouille d'un homme, un cratère en calice (forme d'une valeur particulière), certainement de la main du peintre de vases métapontin et décoré d'une scène représentant le rapt du jeune Céphale par Éos, la déesse de l'aube (fig. 9). Ce groupe réapparaît autour de la déesse étrusque correspondante, Thesan, au sommet d'un haut candélabre en bronze de fabrication volsque inséré dans la seconde tombe, qui contient la dépouille d'une femme (fig. 10, BOtTini, 1985 et 1990).

On peut probablement retrouver une conjonction identique entre privilège sociopolitique et adhésion active à des formes de religiosité liées au destin post-mortem des individus dans le mobilier funéraire, inhabituel, mis au jour au sein de l'habitat de Serra di Vaglio (GRECO, 2013). On y a retrouvé un cratère en cloche du même peintre, quoique de moins bonne qualité, orné du même épisode mythologique. Parmi les autres objets, on distinguera une petite cruche (chous), dont il sera question un peu plus bas, et deux jouets en terre cuite - une panthère (attribut de Dionysos) et une poupée articulée - dont la nature même se prête à une fonction symbolique précise. Dans le mythe, particulièrement cher aux Orphiques, Dionysos enfant est en effet pris au piège, tué et démembré par les Titans, épisode qui en soulignait, à travers le dépassement de la mort, la teneur salvatrice (TORTORELLI GHIDINI, 2000). On voit donc ici entrer en jeu l'une des figures les plus complexes et, à bien des égards, les plus ambiguës du panthéon hellénique : impitoyable envers ceux qui refusaient de reconnaître son pouvoir, comme l'a bien montré Euripide dans Les Bacchantes, Dionysos était en même temps une divinité salvatrice. Sa relation avec la Péninsule dans son ensemble était en outre une réalité bien connue des hommes de l'Antiquité, et ce n'est pas un hasard si Sophocle le définit comme « celui qui protège la glorieuse Italia " (Antigone, v. 1115 et suiv.). D'un point de vue historique, sa connaissance a certainement pu être favorisée en premier lieu par la consommation collective de vin, non pas en tant que telle, mais à travers les pratiques liées à la coutume hellénique. Elles sont documentées par l'adoption de récipients spécifiques comme les cratères, dont la forme même et souvent les dimensions se prêtent à la représentation de scènes complexes, à plusieurs personnages, et qui étaient fréquemment associées à l'ensemble des instruments nécessaires à en préparer l'usage (récipients pour l'eau,
9. Peintre de Pisticci

(Métaponte), cratère à figures rouges avec Éos et Céphale, vers 430 av. notre ère, Ruvo del Monte (tombe 65), Melfi, Museo Archeologico Nazionale del Melfese.

10. Groupe avec Thesan/Éos et Céphale, détail d'un candélabre étrusque (production de Vulci), bronze, $\mathrm{V}^{\mathrm{e}}$ siècle av. notre ère, Ruvo del Monte (tombe 64), Melfi, Museo Archeologico Nazionale del Melfese. 
louches, passoires et râpes en métal). Il pouvait aisément en résulter une identification des participants aux cérémonies aux figures mythologiques du thiasos, satyres et ménades, très souvent représentées sur les céramiques utilisées pour le service de table. De fait, leur image occupe une très large part de la production italiote de céramique à figures rouges, signalant ainsi une notoriété, et donc une acceptation, dont ne bénéficiait aucune autre divinité, y compris les plus importantes.

L'importance du culte dionysiaque ressort cependant aussi d'éléments d'une tout autre nature, comme l'adoption d'au moins une partie des rituels propres aux fêtes des Anthéstéries, ou de rituels qui, du moins, en dérivaient. Il en est ainsi, d'une part, de l'usage consistant à déposer dans la tombe un exemplaire du type particulier de chous que l'on remettait aux jeunes enfants pour marquer leur sortie du premier âge de l'enfance, au deuxième jour des célébrations. Si dans le cas cité précédemment de la sépulture de Serra di Vaglio, il pourrait certes s'agir d'un geste dénué d'une signification aussi précise, dans celui de la sépulture enfantine de Banzi, datable du IV ${ }^{\mathrm{e}}$ siècle, on observe une correspondance parfaite avec la pratique hellénique, consistant précisément à placer un chous dans la tombe des enfants morts avant d'avoir atteint un certain âge. D'autre part, au moins à partir du V viècle, on voit se répandre l'usage consistant à introduire dans le mobilier funéraire des vases de type ordinaire, par exemple des mortiers et surtout des marmites (le plus souvent des lapades), correspondant aux aliments consommés au troisième jour des fêtes, dit des chytroi; il semble qu'il ne s'agisse pas là d'une simple adhésion à un rituel purificatoire d'exorcisme confiné à la sphère funèbre.

Il n'existe toutefois ni preuve concrète, ni mention dans les sources, nous autorisant à penser que les Italiques participaient aussi à d'autres célébrations ou représentations à caractère public plus immersives, comme l'étaient les diverses représentations à travers lesquelles se manifestait la ritualité orphico-dionysiaque (RICCIARDELLI, 2010). Le souvenir d'un passage d'Hérodote (4, 78 et suiv.) offrant le récit d'un épisode de la vie du roi scythe Skyles, nous incite à la prudence : il fut en effet renversé par ses sujets pour s'être fait initier aux mystères dionysiaques, autrement dit pour s'être laissé aller à des comportements assurément destinés à ne pas passer inaperçus. Toutefois, on ne peut pas non plus négliger l'existence d'une catégorie tout à fait particulière de représentations dépeintes sur les céramiques à figures rouges citées plus haut, rassemblées sous le nom de "scènes phlyaques ". Nombreuses en particulier durant la première moitié du $\mathrm{IV}^{\mathrm{e}}$ siècle et sans aucun doute répandues justement dans les centres indigènes, elles témoignent à tout le moins d'un certain intérêt pour des spectacles mis en scène sur des plateaux éphémères, des farces du type de celles que Platon condamnait: "Ne vous imaginez pas que nous vous permettrons de venir si facilement chez nous planter sur la place publique vos tréteaux, et y produire vos acteurs aux belles voix, qui sonnent plus haut que les nôtres ${ }^{12}$."

Il demeure certes difficile d'en imaginer le contexte précis, mais il faut peut-être le mettre en relation avec des fêtes populaires. Avec toute la prudence requise, Carmela Roscino a ainsi émis l'hypothèse selon laquelle ces représentations étaient organisées dans la chôra, lieu dévolu aux rencontres entre les Grecs et les Italiques (ROSCINO, 2012).

On se trouve ici, comme on a déjà eu l'occasion de l'évoquer, en présence de la question véritablement cruciale de la communication entre les uns et les autres. La dimension linguistique de la question, qui n'a d'ailleurs rien de négligeable, dans la mesure où il existait même chez les populations indigènes, et en particulier entre les Apuliens et les Italiques au sens strict, une différence d'idiomes aussi considérable que celle qui les séparait des Grecs, n'est naturellement pas la seule à considérer. Son aspect le plus intéressant réside précisément dans la possibilité de partager des idées, souvent exprimées sous forme de récits mythologiques. En archéologie, 


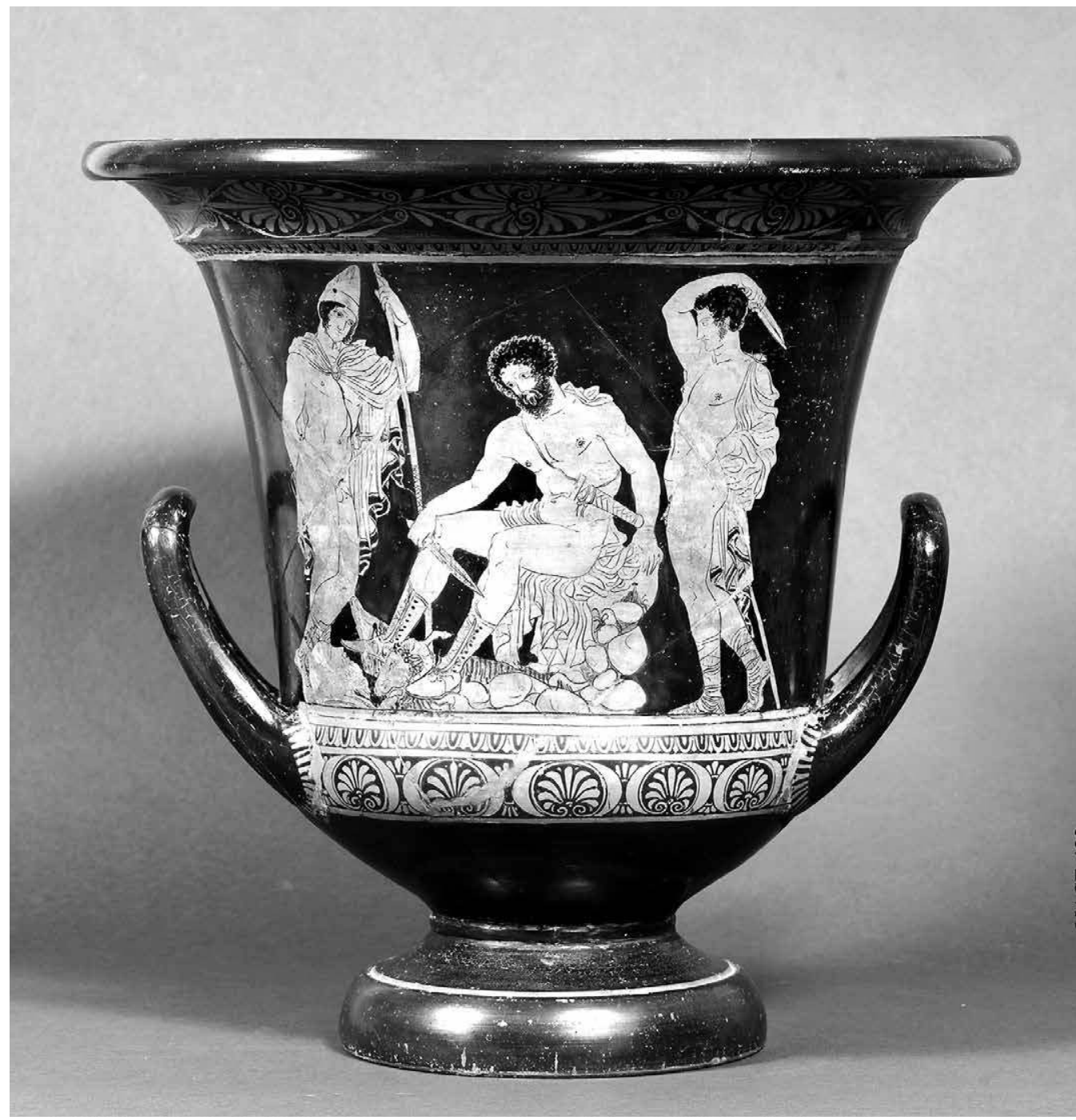

11. Peintre de Dolon

(Métaponte), cratère en calice

avec Ulysse

invoquant les mânes de Tirésias (face A), vers 390

av. notre ère,

découvert à

Pisticci, Paris,

BnF, département

des Monnaies,

Médailles et Antiques.

cette question se pose dans les termes d'un problème très débattu, celui du rapport entre, d'un côté, des commanditaires aux formations culturelles variées, membres de communautés établies loin des poleis, et, de l'autre, des céramistes et des peintres grecs, lorsque des images peintes sur des vases (de formes et de dimensions particulières) trahissent une dépendance étroite et directe envers des textes littéraires et théâtraux selon toute vraisemblance ignorés de la plupart desdits commanditaires.

Suivant la conclusion de la récente analyse du cratère du Peintre de Dolon, il semble difficile de ne pas souscrire à l'hypothèse selon laquelle il aurait existé ce que l'on pourrait aujourd'hui appeler des médiateurs culturels. Peint par l'un des plus grands peintres de vases métapontins, actif durant les premières années du IV siècle, certainement découvert sur le site indigène de Pisticci, il représente une scène très rare, la descente (nekyia) d'Ulysse aux Enfers (fig. 11), dont on a proposé une interprétation complexe suggérant une référence à Pythagore (BOTTINI, 2012 ; GIULIANI, 1995 ; TAPLIN, 2007, p. 21 et suiv., sur des positions contraires). 
Dans ce cas précis, les médiateurs seraient les adeptes eux-mêmes, personnages clefs non seulement pour la mise en circulation de textes ou en tout cas d'un corpus de croyances, du moins au sein des groupes dominants concernés, mais encore (et en l'occurrence surtout) pour l'institution d'un rapport direct avec les artisans du kerameikos urbain, assurément bien plus problématique dans d'autres situations. Une preuve certaine de la présence de cercles initiatiques dans le Métaponte de la fin du V viècle nous est fournie par la célèbre tombe dite " de l'œuf d'Hélène ». Elle fut découverte dans une partie de la chôra qui était peut-être le lieu de résidence de familles appartenant à la classe sociale la plus élevée, mais qui se situait aussi à proximité du centre indigène en question (BOTTINI, 1988 et 1992, p. 64 et suiv.).

En outre, des cas comme celui de la tombe du Plongeur, à Poseidonia peuvent aussi étayer cette thèse : la scène qui donne son nom à cette sépulture, avec son personnage descendant vers l'eau comme vers une libération, depuis un point indéfini, bien plus élevé que le " plongeoir » représentant probablement la limite de l'Océan, est en effet sans aucun équivalent iconographique et chargée d'une signification métaphorique qui renvoie précisément au langage initiatique des célèbres "lamelles" orphiques (BOTTINI, 2016). Ainsi faudrait-il postuler, dans ce type de cas, pouvant prendre des formes diverses mais soulevant les mêmes doutes, l'existence d'œuvres à l'orientation peu banale au regard de l'activité des artisans impliqués dans leur exécution, y compris au sein du même corps civique. 


\section{Notes}

1. Il faut par conséquent faire preuve d'équilibre et de sens critique : de petits bronzes votifs isolés peuvent par exemple attester, plutôt que l'existence de sanctuaires à proprement parler, l'exercice de formes de culte célébrées au long de voies de circulation (BOTTINI, MARCHI, 2016, p. 77).

2. www.regione.vda.it, Aosta, Saint-Martin-de-Corléans, Parco archeologico e Museo.

3. Voir aussi le débat sur la présence de femmes indigènes, intégrées à tel ou tel titre dans les communautés coloniales, et par exemple, à propos de Pithekoussai : GUZZO, 2011, p. 85 ; GUZZO, 2014, contra KELLEY, 2012.

4. La contribution de Giovanni Mastronuzzi, même si elle se révèle parfois trop peu sélective, est de ce point de vue très utile : MASTRONUZZI, 2005.

5. Russo, 2004 ; il vaut la peine de souligner que c'est celle d'Agéladas qui nous a transmis l'image la plus poignante de la condition des femmes apuliennes victimes de ces conflits, et considérées comme des prises de guerre, au même titre que les chevaux, voir Pausanias, $\mathrm{X}$, 10,6 .

6. On a par ailleurs proposé d'établir un lien entre certains des cippes découverts à cet endroit et les tetragonoi lithoi métapontins dont il sera à nouveau question plus loin.

7. Templum tribus modis dicitur, ab natura, ab auspicando, a similitudine: a natura in caelo, ad auspiciis in terra, a similitudine sub terra, Varron, La langue latine, VII, 6, traduction de Désiré Nisard (Paris, 1875).

8. Traité entre Sybaris et les Serdaioi, Olympie, SEG XXII, 336, dernier quart du $\mathrm{VI}^{\mathrm{e}}$ siècle, musée d'Olympie, inv. B 4750 : BROUSSEAU, 2010.

9. Romaniello, 2011 ; fig. 6 [statue] ; fig. 4 [modèles], planche en couleur non numérotée.

10. Conformément au rituel édicté par la table iguvienne IIa, 15 et suiv. : PROSDOCIMI, 1978, p. 713 et suiv., et repris dans plusieurs contextes archéologiques : DE GROSSI, MINNITI, 2000, en particulier en Lucanie, à Torre di Satriano: OsANNA, SICA, 2005, p. 436.

11. Des couples de figures, homme et femme côte à côte, recelant une signification érotique implicite.

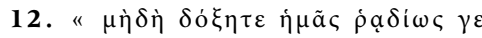

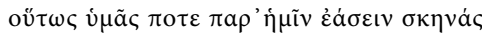

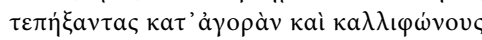

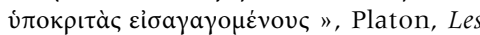
Lois, VII, 817c, traduction de Luc Brisson et Jean-François Pradeau (Paris, 2006).

\section{Bibliographie}

- Armi..., 1993 : Armi. Gli strumenti della guerra in Lucania, Angelo Bottini (dir.), cat. exp. (Melfi, Museo archeologico nazionale del Melfese, 1993), Bari, 1993. - The Art of Italic Peoples..., 1993 : The Art of Italic Peoples from 3000 to 300 B. C. [Swiss Collections], cat. exp. (Genève, musée Rath, 1993-1994/Paris, Mona Bismarck Foundation, 1994), Naples, 1993.

- BARRA BAGNASCO, 1997 : Marcella Barra Bagnasco (dir.), Pomarico Vecchio, I. Abitato mura necropoli materiali, Galatina, 1997.

- BARRA BAgnasco, 2008 : Marcella Barra Bagnasco, "L'area sacra di Fontana Bona di Ruoti: aspetti della religiosità lucana ", dans Alfonsina Russo, Helga Di Giuseppe (dir.), Felicitas Temporum. Dalla terra alle genti: la Basilicata settentrionale tra archeologia e storia, Potenza/Rome, 2008, p. 177-203.

- BATTILORO, OSANNA, 2011 : Ilaria Battiloro, Massimo Osanna (dir.), Brateís Datas. Pratiche rituali, votivi e strumenti del culto dai santuari della Lucania antica, actes de colloque (Matera, 2010, ), Venosa, 2011. - Bertesago, Garaffa, 2015 : Silvia Martina Bertesago, Valentina Garaffa, L'area sacra di Grotte delle Fontanelle a Garaguso. I depositi votivi in proprietà Autera e Altieri, Venosa, 2015.

- BIANCO, 2003 : Salvatore Bianco, "Il santuario di Chiaromonte ", dans Le sacre acque..., 2003, p. 73-80.

- Bottini, 1985 : Angelo Bottini, « Due crateri protoitalioti dal Melfese ", dans Bollettino d'Arte, 30, 1985, p. 55-60.

- BotTini, 1988 : Angelo Bottini, « Elena in Occidente: una tomba dalla chôra metapontina ", dans Bollettino d'Arte, 50-51, 1988, p. 1-18.

- Bottini, 1992 : Angelo Bottini, Archeologia della salvezza, Milan, 1992.

- Bottini, 1999 : Angelo Bottini, "Il candelabro etrusco di Ruvo del Monte", dans Bollettino d'Arte,59, 1999, p. 1-14.

- BotTini, 2012 : Angelo Bottini, " Tiresia e Pitagora fra Greci e Italici: la nekyia del Pittore di Dolone ", dans Mélanges de l'École française de Rome - Antiquité (MEFRA), 124, 2012, 2, p. 461-474.
- Bottini, 2016 : Angelo Bottini, "La Tomba del Tuffatore ", dans Forma urbis, 21, 2016, 11, p. 14-19.

- Bottini, Fresa, TAgliente, 1990 : Angelo Bottini, Maria Pia Fresa, Marcello Tagliente, "L'evoluzione della struttura di un centro daunio fra VII e III secolo: l'esempio di Forentum ", dans Marcello Tagliente (dir.), Italici in Magna Grecia: lingua, insediamenti, strutture, Venosa, 1990, p. 233-264.

- BotTinI, GUzzo, 1992 : Angelo Bottini, Pier Giovanni Guzzo, "Busti fittili da Lavello ", dans Bollettino d'Arte, 72, 1992 , p. 3-10.

- Bottini, LeCCE, 2013 : Angelo Bottini, Lucia Lecce, "La mesogaia lucana e il caso di Pisticci ", dans Luigi Todisco (dir.), La comunicazione verbale tra Greci e indigeni in Apulia nel V-IV secolo a.C.: quali elementi?, actes de colloque (Bari, Seminario di studi linguistici, archeologici e storici, Università degli studi Aldo Moro, 2012), Naples, 2013, p. 45-60.

- BOtTINI, MARCHI, 2016 : Angelo Bottini, Maria Luisa Marchi, Identità e conflitti tra Daunia e Lucania preromane, Pise, 2016. - BotTini, RAININI, Colazzo, 1976 : Angelo Bottini, Ivan Rainini, Stefania Colazzo, « Valle d'Ansanto - Rocca S. Felice, il deposito votivo del santuario di Mefite" ", dans Notizie degli Scavi di Antichità, 1976, p. 359-524.

- Bottini, TAgLiente, Fresa, 1991 : Angelo Bottini, Marcello Tagliente, Maria Pia Fresa, "Relazione sull'area lucana e sul santuario di Lavello ", dans Joseph Mertens, Roger Lambrechts (dir.), Comunità indigene e romanizzazione nell'Italia centro-meridionale, IV - III sec. a.C., actes du colloque (Rome, Institut historique belge de Rome, 1990), Bruxelles/Rome, 1991, p. 93-104.

- Brousseau, 2010 : Louis Brousseau, "Le monnayage des Serdaioi revisité", dans Revue Numismatique, 6, 166, 2010, p. 257-285.

- CARANDini, 2006 : Andrea Carandini, Remo e Romolo. Dai rioni dei Quiriti alla città dei Romani (775/750 - 700/675 a.C.), Turin, 2006.

- CARTER, 2006 : Joseph C. Carter, "The Greek Identity at Metaponto ", dans Kathryn Lomas (dir.), Greek Identity in the Western Mediterranean:Papers in Honour of Brian Brian Shefton, Leiden/Boston, 2006, p. 363-390.

- CAzAnove, 2009 : Olivier de Cazanove, "Luoghi di culto lucani a pianta centrale quadrata ", dans Siris, 10, 2009, p. 131141. 
- Cazanove, 2011: Olivier de Cazanove, "Quadro concettuale, quadro materiale delle pratiche religiose lucane. Per una revisione dei dati ", dans BATTILORO, OSANNA, 2011, p. 295-310.

- CIPRIANI, 1996 : Marina Cipriani, « Prime presenza italiche organizzate alle porte di Poseidonia ", dans Poseidonia e i Lucani, cat. exp. (Paestum, Museo archeologico nazionale, 1996), Marina Cipriani, Fausto Longo (dir.), Naples, 1996, p. 119-158.

- Coppola et al., 2008 : Donato Coppola, Martine Denoyelle, Martine Dewailly, Ivana Fusco, Sebastien Lepetz, Alessandro Quercia, William van Andringa, Thierry van Compernolle, Stéphane Verger, "La grotte de Santa Maria di Agnano (Ostuni) et ses abords : à propos des critères d'identification d'un sanctuaire messapien ", dans Xavier Dupré Raventós, Sergio Ribichini, Stéphane Verger (dir.), Saturnia Tellus: definizioni dello spazio consacrato in ambiente etrusco, italico, fenicio-punico, iberico e celtico, actes du colloque (Rome, 2004), Rome, 2008, p. 201-232.

- CORRENTE et al., 2008 : Marisa Corrente et al., "Prima e dopo Roma. Sostrati formativi e profilo culturale della Daunia alla luce delle recenti attività di scavo della Soprintendenza per i Beni Archelogici della Puglia ", dans Armando Gravina (dir.), Atti del $28^{\circ}$ Convegno nazionale sulla Preistoria, Protostoria, Storia della Daunia, actes de colloque (San Severo, 2007), San Severo, 2008, p. 375-404.

- D'Alessio, 2013 : Maria Teresa D'Alessio, "Riti e miti di fondazione nell'Italia antica. Riflessioni sui luoghi di Roma ", dans Gilda Bartoloni, Laura Maria Michetti (dir.), Mura di legno, mura di terra, mura di pietra: fortificazioni nel Mediterraneo antico, actes de colloque (Rome, Università degli Studi di Roma "La Sapienza", 2012), Rome, 2013, p. 315-331.

- D'ANDRIA, MASTRONUZZI, 2008 : Francesco D'Andria, Giovanni Mastronuzzi, "Cippi e stele nei contesti cultuali della Messapia ", dans Giovanna Greco, Bianca Ferrara (dir.), Dono agli dei. Il sistema dei doni votivi nei santuari, actes de colloque (Naples, Ateneo federiciano, 2006), Naples, 2008, p. 223-240.

- De Grossi, Minniti, 2000 : Jacopo De Grossi, Claudia Minniti, "Le sepolture di cani della necropoli di età imperiale di Fidene - via Radicofani (Roma): alcune considerazioni sul loro seppellimento nell'antichità ", dans Atti del secondo convegno di archeozoologia, actes de colloque (Asti, 1997), Forlì, 2000, p. 367-398.
- D’ERCole, 1990 : Maria Cecilia D’Ercole, La stipe votiva del Belvedere a Lucera, Rome, 1990.

- De Siena, 1998 : Antonio De Siena, "Metaponto: problemi urbanistici e scoperte recenti ", dans Siritide e Metapontino. Storie di due territori coloniali, actes de colloque (Policoro, Centre Jean-Bérard, Fondazione Paestum, 1991), Naples/Paestum, 1998, p. 141-170.

- De Siena, 2014 : Antonio De Siena, " La Puglia ", dans Da Italìa a Italia. Le radici di un'identità, atti del cinquantunesimo Convegno di studi sulla Magna Grecia, actes de colloque (Taranto, Istituto per la storia e l'archeologia della Magna Grecia, 2011), Taranto, 2014, p. 451-495.

- De Simone, 1988 : Carlo De Simone, " Iscrizioni messapiche della Grotta della Poesia (Melendugno, Lecce) ", dans Annali della Scuola Normale Superiore di Pisa, 18, 2, 1988, p. 325-415.

- Di FAzIo 2009: Massimiliano di Fazio, Morte e pianto rituale nell'Italia antica. Il caso dell'askos "Catarinella", dans Maurizio Harari, Silvia Paltineri, Mirella T. A. Robino (dir.), Icone del mondo antico.Un seminario di storia delle immagini, actes du colloque (Pavia, Collegio Ghislieri, 2005), Rome, 2009, p. 205-213.

- FAlasca, 2002 : Giovanna Falasca, " Mefitis, divinità osca delle acque (ovvero della mediazione) ", dans Eutopia, 2, 2 , 2002, p. 7-56.

- FERNÁNDEZ-GÖTZ, 2016 : Manuel Fernández-Götz, " The Power of the Past: Ancestral Cult and Collective Memory in the Central European Iron Age ", dans Valeriu Sîrbu, Miloš Jevtić, Katarina Dmitrović, Marija Ljuština (dir.), Funerary Practices $d u$ ring the Bronze and Iron Ages in Central and Southeast Europe, actes de colloque (Čačak (Serbie), $14^{\text {th }}$ International Colloquium of Funerary Archaeology,2015) Beograd/ Čačak, 2016, p. 165-178.

- Galeandro, Palmentola, 2013 : Fabio Galeandro, Paola Palmentola, " Gli scavi della Scuola di Specializzazione in Beni Archeologici dell'Università di Bari sull'acropoli di Monte Sannace (19942001) ", dans Epigrafia e territorio. Politica e società. Temi di antichità romane, IX, Bari, 2013, p. 31-109.

- GALIOto, 2011 : Giusj Galioto, "Offerte votive e aspetti cultuali ", dans BATTILORO, OSANNA, 2011, p. 139-155.

- GiUliani, 1995 : Luca Giuliani, Tragik, Trauer und Trost. Bildervasen für eine apulische Totenfeier, Berlin, 1995.

- GRECO, 2011: Giovanna Greco, "Acroteri e sistemi decorativi per tetti di età arcaica nel sito indigeno di Vaglio di Basilicata ", dans Carlo Rescigno, Patricia S. Lulof (dir.), Deliciae Fictiles IV: Architectural Terracottas in Ancient Italy. Images of Gods, Monsters and Heroes, actes de colloque (Rome, Museo Nazionale Etrusco di Villa Giulia/Royal Netherlands Institute/ Syracuse, Museo Archeologico Regionale Paolo Orsi, 2009), Oxford/Oakville, 2011, p. 359-377.

- GRECO, 2013 : Giovanna Greco, "Serra di Vaglio. La tomba 68 ", in Segni del potere..., 2013, p. 304-312.

- GRECO, 2016 : Emanuele Greco, "Su alcune analogie (strutturali?) nell'organizzazione dello spazio: il caso delle città achee ", dans Lieve Donnelan, Valentino Nizzo, Gert-Jan Burgers (dir.), Conceptualising Early Colonisation, actes de colloque (Rome, Institut historique belge de Rome, 2012), Bruxelles, 2016, p. 197206.

- GUALTIERI, FRACCHIA, 1990 : Maurizio Gualtieri, Helena Fracchia, Roccagloriosa I. L'abitato: scavo e ricognizione topografica (1976-1986), Naples, 1990.

- Guzzo, 2011 : Pier Giovanni Guzzo, Fondazioni greche. L'Italia meridionale e la Sicilia (VIII e VII sec. a. C.), Rome, 2011.

- Guzzo, 2014 : Pier Giovanni Guzzo, " Dalle fibule all'identità? Il caso di Pithecusa ", dans Giovanna Greco, Bianca Ferrara (dir.), Segni di appartenenza e identità di comunità nel mondo indigeno, actes de colloque (Naples, Università degli Studi di Napoli Federico II, 2012, ), Naples, 2014 , p. 75-87.

- HERRING, 2007 : Edward Herring, " Priestesses in Puglia? An Archaeological Perspective on the Messapic Tabara Inscriptions ", dans Kathryn Lomas, Ruth D. Whitehouse, John B. Wilkins (dir.), Literacy and the State in the Ancient Mediterranean, actes de séminaire (Londres, Institute of classical studies/Institute of archaeology, University College, 20032004), Londres, 2007, p. 129-148.

- HINZ, 1998 : Valentina Hinz, Der Kult von Demeter und Kore auf Sizilien und in der Magna Graecia, Wiesbaden, 1998.

- Holloway, 1970 : R. Ross Holloway, Satrianum. The Archaeological Investigations Conducted by Brown University in 1966 and 1967, Providence, 1970.

- IANNELLI, SABbione, 2014 : Maria Teresa Iannelli, Claudio Sabbione (dir.), Le spose e gli eroi. Offerte in bronzo e in ferro dai santuari della Calabria Greca, Vibo Valentia, 2014 
- Kelley, 2012 : Olivia Kelley, « Beyond Intermarriage: the Role of the Indigenous Italic Population at Pithekoussai ", dans Oxford Journal of Archaeology, 31, 3, 2012, p. 245-260.

- KLEIBRINK MAASKANT, 2003 : Marianne Kleibrink Maaskant, Dalla lana all'acqua. Culto e identità nell'Athenaion di Lagaria, Francavilla Marittima, Rossano, 2003.

- KLEIBRINK, WEISTRA, 2013 : Marianne Kleibrink, Elizabeth Weistra, "Una dea della rigenerazione, della fertilità e del matrimonio. Per una ricostruzione della dea precoloniale della Sibaritide ", dans Giorgio Delia, Tullio Masneri (dir.), Sibari. Archeologia, storia, metafora, Castrovillari, 2013, p. 35-55.

- LA TORRE, 1996 : Gioacchino Francesco La Torre, "Il sacello tardo-arcaico di Campora S. Giovanni (Cs): relazione preliminare ", dans Attilio Stazio, Stefania Ceccoli (dir.), Eredità della Magna Grecia, actes de colloque ( Taranto, XXXV convegno di studi sulla Magna Grecia, 1995), Taranto, 1996, II, p. 703-722.

- LA TORRe, 2011 : Gioacchino Francesco La Torre, "Le lance di Temesa e le offerte di armi nei santuari di Magna Grecia e Sicilia in epoca arcaica ", dans Quaderni di archeologia, 1, 2011, p. 67-104.

- LAZZARINI, POCETTI, 2001 : Maria Letizia Lazzarini, Paolo Poccetti, Il mondo enotrio tra vi e v secolo a.C., 1, 2. L'iscrizione paleoitalica da Tortora, actes de séminaire (Naples, "Il sistema mediterraneo: radici storiche e culturali, specificità nazionali", 1996-1998), Maurizio Bugno, Concetta Masseria (éd.), Naples, 2001.

- LeONe et al., 2015 : Marcella Leone, Priscilla Munzi, Claude Pouzadoux, Nicolas Garnier, Martine Leguilloux, Italo M. Muntoni, " Measure the Sacred at Arpi: the Share of Food and Drink in Two Votive Deposits ", dans Proceedings of 1st International Conference on Metrology for Archaeology, actes de colloque (Benevento, 2015), en ligne : http://193.206.108.81/ metroarcheo2016/2015/files/MetroArcheo2015_Proceedings.pdf, p. 425-430.

- LO PORTO, 1991 : Felice Gino Lo Porto, Timmari. L'abitato, le necropoli, la stipe votiva, Rome, 1991.

- LO PORTO, RANALDI, 1990 : Felice Gino Lo Porto, Francesco Ranaldi, Le "lastre dei cavalieri" di Serra di Vaglio (Monumenti antichi dei Lincei, serie misc. III, 6), Rome, 1990.

- MARCHeSINI, 2013 : Simona Marchesini, "Quali lingue, quali popoli nell'Apulia di v e IV secolo a.C. ", dans La comunicazione verbale tra Greci e indigeni in Apulia nel $V$-IV secolo a.C.: quali elementi?, atti del seminario di studi linguistici, archelogici e storici, actes du colloque (Bari, Università degli studi Aldo Moro, 2012), Naples, 2013, p. 19-33.

- MASSERIA, 1999 : Concetta Masseria, " "Et venerem et proelia destinat..." (Hor. Carm., III, 13, 5) Riti di passaggo in un santuario di Banzi ", dans Ostraka. Rivista di Antichità, 8, 2, 1999, p. 469-490.

- MASSERIA, 2000 : Concetta Masseria, I santuari indigeni della Basilicata. Forme insediative e strutture del sacro, Naples, 2000. - MASTRONUZZI, 2005 : Giovanni Mastronuzzi, Repertorio dei contesti cultuali indigeni in Italia meridionale, 1. Età arcaica, Bari, 2005.

- MASTRONUZZI, 2013 : Giovanni Mastronuzzi, Il luogo di culto di Monte Papalucio ad Oria, 1. La fase arcaica, Bari, 2013.

- MAZZEI, 2010 : Marina Mazzei, I Dauni. Archeologia dal IX al V secolo a.C., Foggia, 2010.

- MeLe, 1981 : Alfonso Mele, «Il pitagorismo e le popolazioni anelleniche d'Italia ", dans AION: quaderno. ArchStAnt, 3, 1981, p. 61-96.

- Mele, 2000 : Alfonso Mele, "Megale hellas e Pitagorismo ", dans TORTORELLI GHIDINI, STORCHI, VISCONTI, 2000, p. 297-333. - Montanaro, 2010 : Andrea Celestino Montanaro, " La ceramica a figure nere in area apula. Produzioni, diffusione e contesti ", dans Mediterranea, 7, 2010 , p. 203-268.

- MORET, 2014 : Jean-Marc Moret, I marmi di Garaguso. Vittorio Di Cicco e l'imbroglio della loro scoperta, Domingo Gasparro (coll.), Venosa, 2014.

- Mutino, 2012 : Sabrina Mutino, "Strutture palaziali a Lavello tra VI e v sec. a.C. ", dans Massimo Osanna, Vincenzo Capozzoli (dir.), Lo spazio del potere, II. Nuove ricerche nell'area dell'anaktoron di Torre di Satriano, actes de colloque (Tito, terzo e quarto convegno di studi su Torre di Satriano, 2009-2010), Venosa, 2012, p. 187-203.

- OsAnNA, 2008 : Massimo Osanna, "Monumenti, commemorazione e memoria in Daunia: la collina del Serpente di Ascoli Satriano tra età arcaica e conquista romana ", dans Giuliano Volpe, Maria Josè Strazzulla, Danilo Leone (dir.), Storia e archeologia della Daunia, in ricordo di Marina Mazzei, actes de colloque (Foggia, 2005), Bari, 2008, p. 149-170.

- OSANNA, SICA, 2005 : Massimo Osanna, Maria Maddalena Sica (dir.), Torre di Satriano, I. Il santuario lucano, Venosa, 2005.
- PACCIARElli, 2016 : Marco Pacciarelli, "Forme di insediamento del Primo Ferro in Calabria ", dans Lieve Donnellan, Valentino Nizzo, Gert-Jan Burgers (dir.), Contexts of Early Colonosation, actes du colloque (Rome, Royal Netherlands Institute), I, Rome, 2016, p. 177-181.

- PAoletti, 2014 : Maurizio Paoletti, « La necropoli enotria di Macchiabate, Lagaria e la "dea di Sibari" ", dans Paolo Brocato (dir.), Studi sulla necropoli di Macchiabate a Francavilla Marittima (Cs) e sui territori limitrofi, Arcavacata di Rende, 2014, p. 7-21. - Poccetti, 1996 : Paolo Poccetti, " Culti delle acque e stadi della vita muliebre: dottrine misteriche e fondo religioso italico nella tavola osca di Agnone ", dans Loretta Del Tutto Palma (dir.), La tavola di Agnone nel contesto italico, actes du colloque (Agnone, 1994), Florence, 1996, p. 219-241.

- Poccetti, 2000 : Paolo Poccetti, "La diffusione di dottrine misteriche e sapienziali nelle culture indigene dell'Italia antica: appunti per un dossier ", dans TORTORELLI GHIDINI, STORCHI, VISCONTI, 2000, p. 93-126.

- Poccetti, 2008a : Paolo Poccetti, « Un Case Study per l'identificazione di un santuario messapico: il materiale epigrafico dalla grotta di S. Maria di Agnano (Ostuni, Brindisi) ", dans Sergio Ribichini, Stéphane Verger (dir.), Saturnia Tellus: definizioni dello spazio consacrato in ambiente etrusco, italico, fenicio-punico, iberico e celtico, actes du colloque (Rome, 2004), Rome, 2008, p. 233-249.

- Poccetti, 2008b : Paolo Poccetti, « Mefitis rivisitata (vent'anni dopo... e oltre, con prolegomeni e epilegomeni minini) ", dans Alfonso Mele (dir.), Il culto della dea Mefite e la Valle d'Ansanto: ricerche su un giaciamento archeologico e culturale dei Samnites Hirpini, actes de colloque (Avellino-Villamaina/Rocca San Felice, 2002), Avellino, 2008, p. 139-179.

- PROsDocimi, 1978 : Aldo Luigi Prosdocimi, "L'umbro ", dans Lingue e dialetti dell'Italia antica (Popoli e civiltà dell'Italia antica, VI), Rome, 1978, p. 585-787.

- RICCIARDELLI, 2010 : Gabriella Ricciardelli, "L'elemento spettacolare nel rito orfico-dionisiaco ", dans La Parola del Passato, 65, 2010, p. 98-126.

- Romaniello, 2011 : Mara Romaniello, " (San Chirico Nuovo: l'area di culto in località Pila). Offerte votive e aspetti cultuali ", dans BATTILORO, OSANNA, 2011 , p. 151-171.

- Roscino, 2009 : Carmela Roscino, "Con gli occhi dell'altro: gli esordi della 
rappresentazione di Italici sui vasi lucani e apuli a figure rosse tra la fine del v e gli inizi del IV secolo a.C. ", dans Ostraka. Rivista di Antichità, 18, 2, 2009, p. 483-507. - Roscino, 2012 : Carmela Roscino, " Commedia e farsa ", dans Lugi Todisco (dir.), La ceramica a figure rosse della Magna Grecia e della Sicilia, Rome, 2012, II, p. 287-295.

- Russo, 2004 : Federico Russo, "I donari tarantini a Delfi ", dans Annali della Scuola Normale Superiore di Pisa. Classe di Lettere e Filosofia, 9, 1, 2004, p. 79-102.

- RUTA SERAFINI, SAINATI, 2002 : Angela Ruta Serafini, Camilla Sainati, "Il "caso" Meggiaro: problemi e prospettive "; II. 1. " La nuova scoperta. Il santuario orientale a Meggiaro " dans Este preromana: una città e i suoi santuari, Angela Ruta Serafini (dir.), cat. exp. (Este, Museo Nazionale Atestino nella sede di palazzo Mocenigo, 2002), Trévise, 2002, , p. $216-$ 223.

- Le sacre acque..., 2003 : Le sacre acque. Sorgenti e luoghi del rito nella Basilicata antica, Marcello Tagliente (dir.), cat. exp.(Potenza, Museo Provinciale, 2003-2004), Lavello, 2003.

- Segni del potere..., 2013 : Segni del potere. Oggetti di lusso dal Mediterraneo nell'Appennino lucano di età arcaica, Massimo Osanna, Mariasilvia Vullo (dir.), cat. exp. (Potenza, Museo archeologico nazionale della Basilicata "Dinu Adamesteanu", 2013), Venosa, 2013.

- SETARI, 2009 : Elisabetta Setari, « Terrecotte architettoniche da Braida di Vaglio: alcune considerazioni ", dans Ostraka. Rivista di Antichità, 18, 1, 2009, p. 249-275. - SPATAFORA, 2006 : Francesca Spatafora, "Vincitori e vinti: sulla deposizione di armi e armature nella Sicilia di età arcaica ", dans Carmine Ampolo, Maria Adelaide Vaggioli (dir.), Guerra e pace in Sicilia e nel Mediterraneo antico (VIII-III sec. a.C.). Arte, prassi e teoria della pace e della guerra, actes de colloque (Erice [Trapani], 2003) Pise, 2006, I, p. 215-226.

- SPATAFORA, 2016 : Francesca Spatafora (dir.), Il Thesmophorion di Entella. Scavi in Contrada Petraro, Pise, 2016.

- TAgliente, 1985/1986 : Marcello Tagliente, "I Signori dei Cavalli nella Daunia di età arcaica ", dans Annali Facoltà di Lettere e Filosofia, Università di Perugia, XXXIII 1 (studi classici), 1985/1986, p. 305-321.

- TAgLiente, 1991 : Marcello Tagliente, " A. L'acropoli di Lavello e i suoi monumenti ", dans Angelo Bottini, Maria Pia Fresa (dir.), Forentum II - L'acropoli in età classica, Venosa, 1991, p. 17-26.
- TAPLIN, 2007 : Oliver Taplin, Pots and Plays. Interactions between Tragedy and Greek Vase-painting of the Fourth Century B. C., Los Angeles, 2007.

- TORTORELLI GHIDINI, 2000 : Marisa Tortorelli Ghidini, " Da Orfeo agli Orfici ", dans TORTORELLI GHIDINI, STORCHI, VISCONTI, 2000, p. 11-41.

- TORTORELLI GHIDINI, STORCHI, VISCONTI, 2000 : Marisa Tortorelli Ghidini, Alfredo Storchi, Marino Amedeo Visconti (dir.), Tra Orfeo e Pitagora-origini e incontri di culture nell'antichità, actes de séminaire (Naples, 1996-1998), Naples, 2000. 